\title{
RELATING NOTIONS OF CONVERGENCE IN GEOMETRIC ANALYSIS
}

\author{
BRIAN ALLEN AND CHRISTINA SORMANI
}

\begin{abstract}
We relate $L^{p}$ convergence of metric tensors or volume convergence to a given smooth metric to intrinsic flat and Gromov-Hausdorff convergence for sequences of Riemannian manifolds. We present many examples of sequences of conformal metrics which demonstrate that these notions of convergence do not agree in general even when the sequence is conformal, $g_{j}=f_{j}^{2} g_{0}$, to a fixed manifold. We then prove a theorem demonstrating that when sequences of metric tensors on a fixed manifold $M$ are bounded, $(1-1 / j) g_{0} \leq g_{j} \leq K g_{0}$, and either the volumes converge, $\operatorname{Vol}_{j}(M) \rightarrow \operatorname{Vol}_{0}(M)$, or the metric tensors converge in the $L^{p}$ sense, then the Riemannian manifolds $\left(M, g_{j}\right)$ converge in the measured Gromov-Hausdorff and volume preserving intrinsic flat sense to $\left(M, g_{0}\right)$.
\end{abstract}

\section{INTRODUCTION}

There are many settings in Riemannian geometry where one must examine a sequence of Riemannian manifolds and prove that they converge in some sense to a limit space. These situations arise when one is seeking a canonical metric in a given class, or examining how manifolds evolve under flows, or studying the stability of a rigidity theorem. When the convergence cannot be proven to be smooth, because known examples exist which do not converge smoothly, then one can still hope to prove convergence in some weaker sense. Analysts will immediately be drawn to consider notions like Lesbegue $\left(L^{p}\right)$ convergence of metric tensors. Geometers will turn instead to geometric notions like Gromov-Hausdorff $(\mathrm{GH})$ convergence in which distances are controlled but not metric tensors. If controlling distances seems too strong a notion, Geometers turn to Intrinsic Flat $(\mathcal{F})$ convergence, in which only the filling volumes between the spaces and their limits must tend to 0 . Neither GH nor $\mathcal{F}$ convergence imply volumes of the spaces converge, and so geometers also consider the stronger metric measure (See Sturm Stu06a for the best definition of metric measure convergence), measured Gromov-Hausdorff distance $(\mathrm{mGH})$, or volume preserving intrinsic flat $\mathcal{V} \mathcal{F}$ convergences as well. We review all these notions in Section 2 .

C. Sormani was partially supported by NSF DMS 1612049. Some of this work was completed in discussions at the IAS Emerging Topics on Scalar Curvature and Convergence that C. Sormani co-organized with M. Gromov in 2018. 
Understanding compactness properties for sequences of Riemannian manifolds under various geometric conditions is a vast area of research in geometric analysis. Important work where integral bounds on curvature or volume pinching is assumed has been done by Anderson And90, And05, Anderson and Cheeger [AC91, Colding Col97, Col96, Gao Gao90, Petersen and Wei PW97, PW01, Petersen and Sprouse PS98, and Yang Yan92c, Yan92a, Yan92b. In these works the authors have various results showing $C^{0, \alpha}$ (possibly away from singular points) convergence of Riemannian metrics under various integral bounds on curvature or assumptions on volume (See the survey articles of Petersen [Pet97] and Sormani [Sor12] for a broad discussion of convergence theorems). In the case of convergence of conformal Riemannian metrics important related work has been done by Aldana, Carron, and Tapie ACT18, Brendle Bre03, Bonk, Heinonen, and Saksman [BHS08, Chang, Gursky, and Wolff [CGW94, Gursky Gur93, and Wang Wan15] (See the survey article by Chang Cha05] for a broad look at results in conformal geoemtry).

In this paper our goal is to provide hypotheses which can by used to bootstrap from Lebesgue convergence $\left(L^{p}\right)$ or volume convergence to the geometric notions of convergence like $\mathrm{GH}$ and $\mathcal{F}$. Before we state our theorems, we describe the examples we've constructed which provide new insight into the distinctions between these notions of convergence in the conformal setting. We consider $M_{j}=\left(M^{m}, f_{j}^{2} g_{0}\right)$ where $\left(M^{m}, g_{0}\right)$ is either a standard flat torus or a sphere. We begin with Example 3.1 in which the sequence converges in the $L^{p}$ sense to the standard sphere but the GH and $\mathcal{F}$ limit is a cinched sphere. This occurs because Lesbegue convergence cannot see what happens on a set, $S$, of measure 0 (in this case the equator) and yet distance based notions of convergence detect short cuts through $S$ if $g_{j}$ is smaller than the expected $L^{p}$ limit on $S$. To avoid this difficulty of having shorter paths we require $C^{0}$ convergence from below in the rest of our examples and our theorems.

In Examples 3.2- 3.3, we consider conformally flat tori $M_{j}=\left(M^{m}, f_{j}^{2} g_{0}\right)$ with $f_{j} \geq(1-1 / j)$. In Example 3.2 we add bumps to the tori where $f_{j}=K>1$ so that we have $L^{p}$ convergence but not $C^{0}$ convergence. Here we see that $M_{j}$ converge in the $\mathrm{GH}, \mathcal{F}, \mathrm{mGH}$, and $\mathcal{V} \mathcal{F}$ sense to $\left(M, g_{0}\right)$. In Example 3.3 we have $1 \leq f_{j} \leq \sqrt{2}$ and $f_{j} \rightarrow 1$ pointwise on a dense set but no $L^{p}$ convergence. Due to the fact that more and more bumps are appearing increasingly on a finer and finer grid it becomes advantageous to travel along taxi curves and hence we find $\mathrm{GH}$ and $\mathcal{F}$ convergence to a torus with a taxi metric.

In the remaining examples we explore what can happen when the uniform upper bound on the metric is removed. In these examples we see that the particular $p$ for which we have $L^{p}$ bounds and/or convergence of the conformal factor $f_{j}$ plays a crucial role. In Example 3.4 we have a sequence of tori with bumps that grow taller with $j$ while keeping $L^{m}$ convergence of 
$f_{j} \rightarrow 1$ (where $m$ is the dimension of $M^{m}$ ). Here we see that the volumes converge and that we have $\mathrm{mGH}$ and $\mathcal{V F}$ convergence. In Example 3.5 we allow the bumps to grow enough that we do not have $L^{m}$ convergence of $f_{j}$ and the volumes don't converge but both do remain bounded above. In this case we find $\mathrm{mGH}$ and $\mathcal{V F}$ convergence to a flat torus with a flat disk attached so that the boundary of the disk is attached to a point of the flat torus. This shows that the volume convergence assumption is crucial for ruling out bubbling. In Example 3.6 we allow the bumps to grow enough that the $L^{p}$ norm of $f_{j}$ diverges for any $p \geq m$. In this case we see that the sequence does not converge in the GH or $\mathcal{F}$ sense to a compact metric space. This last example illustrates the worst that can happen when we do not have volume convergence. In Example 3.7 we allow the bumps to grow so that we have $L^{m}$ convergence of $f_{j} \rightarrow 1$ and convergence of volume, which is the borderline case between Examples 3.4 and 3.5, and we see that the GH limit is a torus with a line attached. In Example 3.8 we adapt the previous example to allow increasingly many bumps to form while maintaining $L^{m}$ of $f_{j} \rightarrow 1$ and volume convergence so that there is no GH limit.

Despite all these intriguing examples, we are able prove a surprisingly strong theorem about sequences of metrics which are all conformal to the same base manifold:

Theorem 1.1. Suppose we have a sequence of conformal metrics, $f_{j}^{2}(x) g_{0}$, $f_{j}: M \rightarrow(0, \infty)$ continuous, $g_{0}$ smooth, on a compact oriented manifold without boundary, $M$, such that

$$
0<1-1 / j \leq f_{j} \leq K<\infty .
$$

If we have Lebesgue convergence of the conformal factors,

$$
f_{j} \rightarrow 1 \text { in } L^{p}\left(M, g_{0}\right), \quad p \in[1, \infty],
$$

or volume convergence,

$$
\operatorname{Vol}_{g_{j}}(M) \rightarrow \operatorname{Vol}_{g_{0}}(M)
$$

then the sequence of continuous Riemannian manifolds $M_{j}=\left(M, f_{j}^{2}(x) g_{0}\right)$ converges to $M_{0}=\left(M, g_{0}\right)$ in both the measured Gromov-Hausdorff sense and the volume preserving intrinsic flat sense:

$$
M_{j} \stackrel{m G H}{\longrightarrow} M_{0} \text { and } M_{j} \stackrel{\mathcal{V \mathcal { F }}}{\longrightarrow} M_{0} .
$$

Note that Example 3.1 demonstrates that we cannot expect convergence to $M_{0}$ in any sense without $C^{0}$ lower bound in the hypotheses. Example 3.3 demonstrates that we cannot expect convergence to to $M_{0}$ in the $\mathrm{mGH}$ sense if we remove the hypothesis on $L^{p}$ convergence or volume convergence in Theorem 1.1. Example 3.7 and Example 3.8 show that we cannot expect the conclusion of Theorem 1.1 to hold when a uniform upper bound is not assumed.

This conformal theorem is in fact a consequence of the following far more general theorem that we prove within: 
Theorem 1.2. Assume that $g_{j}$ is a sequence of continuous Riemannian metrics and $g_{0}$ is a smooth Riemannian metric defined on the same compact oriented manifold without boundary $M^{m}$ and

$$
(1-1 / j) g_{0}(v, v) \leq g_{j}(v, v) \leq K g_{0}(v \cdot v) \quad \forall v \in T_{p} M .
$$

If we have Lebesgue convergence of the metric tensors,

$$
\int_{M}\left|g_{j}-g_{0}\right|_{g_{0}}^{p} d V_{g_{0}} \rightarrow 0, \quad p \in[1, \infty]
$$

or volume convergence,

$$
\operatorname{Vol}_{g_{j}}(M) \rightarrow \operatorname{Vol}_{g_{0}}(M)
$$

then the sequence of Riemannian manifolds $M_{j}=\left(M, g_{j}\right)$ converges to $M_{0}=\left(M, g_{0}\right)$ in both the measured Gromov-Hausdorff sense and the volume preserving intrinsic flat sense:

$$
M_{j} \stackrel{m G H}{\longrightarrow} M_{0} \text { and } M_{j} \stackrel{\mathcal{V \mathcal { F }}}{\longrightarrow} M_{0} .
$$

In previous work, the authors proved this theorem for sequences of warped Riemannian manifolds [AS19]. We also presented examples of warped product manifolds demonstrating that there are sequences which converge in each sense that don't converge in the other, and that there are sequences which have different limits depending upon the notion of convergence that is considered. The convergence theorem was then applied in $\left[\mathrm{AHP}^{+} 19\right]$ by the first named author with Hernandez-Vazquez, Parise, Payne, and Wang to prove Gromov's Conjecture on the Stability of the Scalar Torus Rigidity Theorem in the warped product setting. The first named author will be applying results from this paper to prove Gromov's Conjecture in the conformal setting. We believe there should be many other applications of this theorem as well.

In Section 2 we review the definitions of Gromov-Hausdorff $(\mathrm{GH})$ and metric measure $(\mathrm{mGH})$ convergence, Intrinsic Flat Convergence $(\mathcal{F})$ and Volume Preserving Intrinsic Flat $(\mathcal{V F})$ convergence and key theorems relating them in the simplified setting where all the spaces are Riemannian manifolds. Theorem 2.3 specializes a result of Gromov Gro81b and HuangLee-Sormani [HLS17] stating that Riemannian manifolds with bi-Lipschitz bounds on their distances have subsequences which converge in the uniform, GH, and $\mathcal{F}$ sense to the same limit space. Theorem 2.4 states that if a sequence of Riemannian manifolds converges in the $\mathrm{GH}$ and $\mathcal{F}$ sense to the same Riemannian limit space, and if the volumes of the manifolds converge to the volume of the limit, then the sequences converge in the $\mathcal{V} \mathcal{F}$ and $\mathrm{mGH}$ sense as well. With this background the reader may proceed to read this paper and apply our results without reading any additional articles on any of these notions of convergence.

In Section 3 we include detailed presentations of the eight examples mentioned above. Some are conformal to a flat torus and the rest are conformal to a standard round sphere. The conformal factors are precisely given and 
the statement of each example is followed by a detailed proof of the claimed convergence and/or lack of convergence for that example.

In Section 4 we give the proof of Theorem 1.2. In particular this involves obtaining estimates on volume and control on distances which leads to completing the proof of the main theorems in subsection 4.4. A key new result is given in Theorem 4.4 which shows that a metric lower bound combined with volume convergence implies pointwise convergence of $d_{j}(p, q) \rightarrow d_{0}(p, q)$ for almost every $(p, q) \in M \times M$. Due to the uniform bounds on the metric assumed in Theorem 1.2 we are then able to show uniform, GH, and $\mathcal{F}$ convergence to a length space by applying a theorem of Huang, Lee, and the second named author in the Appendix of [HLS17]. By combining with the pointwise almost every convergence of distances we are able to conclude that the length space guaranteed by compactness must be the metric respect to the desired background Riemannian metric.

Acknowledgements: We are grateful to Raquel Perales for closely reading this preprint and suggesting improvements particularly to Theorem 4.4. We are also grateful to the other participants at the IAS Emerging Topics on Scalar Curvature and Convergence especially Misha Gromov for inspiring conversations. The second named author would particularly like to thank Alice Chang for finding funding that enabled her to visit IAS and Princeton weekly last year. The first named author would like to thank Ian Adelstein for the invitation to speak at the Filling Volumes, Geodesics, and Intrinsic Flat Convergence conference at Yale University. The first named author would also like to thank Lan-Hsuan Huang and Maree Jaramillo for the invitation to speak at the Spring Eastern Sectional Meeting of the AMS. We would also like to thank the referee for such a careful reading of the paper with such good suggestions for improving the manuscript.

\section{REVIEW}

It is our goal that this paper be easily read by Geometric Analysts who are not necessarily experts in the theory of Metric Spaces and Geometric Measure Theory. We begin by reviewing the fact that $C^{0}$ bounds on metric tensors provide Lipschitz bounds on distance functions: observing that $C^{0}$ Convergence of Riemannian Manifolds implies Gromov Lipschitz Convergence of the Riemannian manifolds viewed as metric spaces. We review the notions of Gromov-Hausdorff $(\mathrm{GH})$ and Intrinsic Flat Convergence $(\mathcal{F})$ in the special setting where we consider only Riemannian manifolds and not the more singular spaces studied by Metric Geometers. We then state and review the key compactness theorem we will be applying to prove our results in this special case where our manifolds are Riemannian. We next review metric measure $(m G H)$ convergence and Volume Preserving Intrinsic Flat $(\mathcal{V F})$ convergence and a key theorem relating these notions in the Riemannian setting. Finally we discuss Lebesgue convergence of metric tensors and prior results relating this notion to $\mathcal{F}$ and $\mathrm{GH}$ convergence. We do not 
attempt to provide a comprehensive review but focus instead only on the results we need in this paper. In particular we apologize for not reviewing the extensive literature on conformal convergence.

2.1. From $C^{0}$ Convergence of Metric Tensors to Lipschitz Convergence of Distances. Recall that a connected continuous Riemannian manifold $(M, g)$ is a metric space $(M, d)$ with a length distance

$$
d_{g}(p, q)=\inf \left\{L_{g}(C): C(0)=p, C(1)=q, \text { piecewise smooth }\right\}
$$

where

$$
L_{g}(C)=\int_{0}^{1} g\left(C^{\prime}(s), C^{\prime}(s)\right)^{1 / 2} d s .
$$

Given bounds on the metric tensor, we have Lipschitz controls on these distances:

Lemma 2.1. If $g_{j}$ and $g_{0}$ are complete continuous Riemannian metrics defined on a connected manifold $M$ so that

$$
g_{j}(v, v) \leq K g_{0}(v, v), \quad \forall v \in T_{p} M
$$

then for $q_{1}, q_{2} \in M$

$$
d_{j}\left(q_{1}, q_{2}\right) \leq K^{1 / 2} d_{g_{0}}\left(q_{1}, q_{2}\right) .
$$

Proof. Let $\gamma$ be a piecewise smooth curve connecting $q_{1}, q_{2} \in M$. By the assumption that $g_{j}(v, v) \leq K g_{0}(v, v)$ we can conclude that

$$
\begin{aligned}
d_{g_{j}}\left(q_{1}, q_{2}\right) & \leq \int_{\gamma}\left(g_{j}\left(\gamma^{\prime}(t), \gamma^{\prime}(t)\right)^{1 / 2} d t\right. \\
& \leq \int_{\gamma}\left(K g_{0}\left(\gamma^{\prime}(t), \gamma^{\prime}(t)\right)\right)^{1 / 2} d t \\
& \leq K^{1 / 2} \int_{\gamma} g_{0}\left(\gamma^{\prime}(t), \gamma^{\prime}(t)\right)^{1 / 2} d t=K^{1 / 2} L_{g_{0}}(\gamma) .
\end{aligned}
$$

By taking the infimum over all curves $\gamma$ we find

$$
d_{g_{j}}\left(q_{1}, q_{2}\right) \leq K^{1 / 2} d_{g_{0}}\left(q_{1}, q_{2}\right) .
$$

Thus we see immediately that two-sided bounds on $g_{j}$ imply bi-Lipschitz bounds on $d_{j}$ :

Lemma 2.2. If $g_{j}$ and $g_{0}$ are complete continuous Riemannian metrics defined on a connected manifold $M$ so that

$$
K_{0} g_{0}(v, v) \leq g_{j}(v, v) \leq K_{1} g_{0}(v, v) \quad \forall v \in T_{p} M
$$

then

$$
K_{0}^{1 / 2} d_{0}\left(q_{1}, q_{2}\right) \leq d_{j}\left(q_{1}, q_{2}\right) \leq K_{1}^{1 / 2} d_{g_{0}}\left(q_{1}, q_{2}\right) .
$$


This can lead to a lot of confusion when people discuss Lipschitz convergence without specifying whether they mean Lipschitz convergence of the metric tensor or Lipschitz convergence of the metric spaces. Gromov defined Lipschitz convergence in Gro81b of metric spaces, $\left(M_{j}, d_{j}\right)$ to $\left(M_{0}, d_{0}\right)$ if there exist bi-Lipschitz maps from $\left(M_{j}, d_{j}\right)$ to $\left(M_{0}, d_{0}\right)$ whose bi-Lipschitz constants converge to 1 . In other words:

$$
C^{0} \text { convergence of }\left(M, g_{j}\right) \rightarrow\left(M, g_{0}\right)
$$

implies

$$
\text { Gromov Lipschitz convergence of }\left(M, d_{j}\right) \rightarrow\left(M, d_{0}\right) \text {. }
$$

A weaker notion of convergence is the uniform convergence of the distance functions as functions $d_{j}: M \times M \rightarrow[0, D]$ where $D$ is a uniform upper bound on the diameter of $\left(M, d_{j}\right)$. We will discuss this uniform convergence more later.

2.2. Gromov-Hausdorff Convergence. The Gromov-Hausdorff (GH) convergence of compact metric spaces was defined by Gromov [Gro81b] as a way of weakening the notion of uniform convergence to sequences of distinct metric spaces. Although it has been defined for metric spaces it has proven to be very useful when studying Riemannian manifolds as well.

Recall that a distance preserving map, $\varphi:(M, d) \rightarrow\left(Z, d_{Z}\right)$, satisfies

$$
d_{Z}\left(\varphi(p), \varphi\left(p^{\prime}\right)\right)=d\left(p, p^{\prime}\right) \quad \forall p, p^{\prime} \in M .
$$

In metric geometry books like [Gro81b] this is called an "isometric embedding" however the notion does not agree with the Riemannian notion of an "isometric embedding". For example, the map from the circle to Euclidean space $F: \mathbb{S}^{1} \rightarrow \mathbb{E}^{2}$, defined by

$$
F(\theta)=(\cos (\theta), \sin (\theta))
$$

is a Riemannian isometric embedding but it is not distance preserving because $d_{\mathbb{S}^{1}}\left(\theta, \theta^{\prime}\right)$ is an arclength along the circle while $d_{\mathbb{E}^{2}}\left(F\left(\theta^{\prime}\right), F\left(\theta^{\prime}\right)\right)$ is the length of a line segment.

The Gromov-Hausdorff (GH) distance between compact metric spaces is then defined

$$
d_{G H}\left(\left(M_{1}, d_{1}\right),\left(M_{2}, d_{2}\right)\right)=\inf d_{H}^{Z}\left(\varphi_{1}\left(M_{1}\right), \varphi_{2}\left(M_{2}\right)\right)
$$

where the infimum is over all compact metric spaces $Z$ and all distance preserving maps $\varphi_{i}: M_{i} \rightarrow Z$ and $d_{H}^{Z}$ is the Hausdorff distance between subsets in $Z$ :

$$
d_{Z}^{H}\left(A_{1}, A_{2}\right)=\inf \left\{r: A_{1} \subset T_{r}\left(A_{2}\right) \text { and } A_{2} \subset T_{r}\left(A_{1}\right)\right\} .
$$

Here $T_{r}(A)$ is the tubular neighborhood of radius $r$ about $A$. So

$$
A_{1} \subset T_{r}\left(A_{2}\right) \Longleftrightarrow \forall x_{1} \in A_{1} \exists x_{2} \in A_{2} \text { s.t. } d_{Z}\left(x_{1}, x_{2}\right)<r .
$$

This then defines

$$
\left(M_{j}, d_{j}\right) \stackrel{\mathrm{GH}}{\longrightarrow}\left(M_{\infty}, d_{\infty}\right) \Longleftrightarrow d_{G H}\left(\left(M_{j}, d_{j}\right),\left(M_{\infty}, d_{\infty}\right)\right) \rightarrow 0 .
$$


This is true iff $\exists$ compact $Z_{j}$ and distance preserving maps, $\varphi_{j}: M_{j} \rightarrow Z_{j}$, and $\varphi_{j}^{\prime}: M_{\infty} \rightarrow Z_{j}$ such that

$$
d_{H}^{Z_{j}}\left(\varphi_{j}\left(M_{j}\right), \varphi_{j}^{\prime}\left(M_{\infty}\right)\right) \rightarrow 0 .
$$

One says that a map, $\psi: M_{1} \rightarrow M_{2}$, is a $\delta$ almost isometry if is it $\delta$-almost distance preserving:

$$
\left|d_{2}\left(\psi(p), \psi\left(p^{\prime}\right)\right)-d_{1}\left(p, p^{\prime}\right)\right|<\delta \quad \forall p, p^{\prime} \in M .
$$

and is $\delta$ almost onto:

$$
M_{2} \subset T_{\delta}\left(\psi\left(M_{1}\right)\right) .
$$

Gromov proved (cf. Corollary 7.3.28 in [BBI01] ) that if there is a $\delta$ almost isometry, $\psi: M_{1} \rightarrow M_{2}$, then

$$
d_{G H}\left(M_{1}, M_{2}\right)<2 \delta .
$$

In this paper we have a fixed background manifold $M$ for the whole sequence, and we will see that having this fixed $M$ allows one to define a sequence of common metric spaces $Z_{j}$ more easily if one has the right hypotheses on the metric tensors. We will also present some examples demonstrating that one may not obtain GH convergence when the hypotheses of our main theorem fail to hold. See the recent work of Aldana, Carron, and Tapie [ACT18] where the authors are able to show GH compactness for sequences of conformal Riemannian manifolds with integral bounds on the scalar curvautre and bounds on the volume.

2.3. Intrinsic Flat Convergence. The intrinsic flat distance $(\mathcal{F})$ defined by Sormani-Wenger in [SW11] is defined for a large class of metric spaces called integral current spaces. In their paper they show that $\mathcal{F}$ is a weaker notion than Gromov Lipschitz convergence that is distinct from GH convergence and can give different limits. In this paper we will explore this further.

As this article is intended for Riemannian geometers, we provide the definition of $\mathcal{F}$ convergence in the setting where the metric spaces are compact oriented Riemannian manifolds, $\left(M, g_{0}\right)$, endowed with distance functions, $d_{j}$, that satisfy

$$
\lambda \geq \frac{d_{j}(p, q)}{d_{0}(p, q)} \geq \frac{1}{\lambda}
$$

where $d_{0}=d_{g_{0}}$ is the length distance associated to $g_{0}$ as above. The distance functions need not arise from Riemannian metrics as long as they satisfy (31). They might for example be the taxi metric on a torus:

$$
\left(\mathbb{T}^{2}, d_{\text {taxi }}\right)=\mathbb{S}^{1} \times_{\text {taxi }} \mathbb{S}^{1}
$$

where

$$
d_{\text {taxi }}\left(p, p^{\prime}\right)=d_{\mathbb{S}^{1}}\left(p_{1}, p_{1}^{\prime}\right)+d_{\mathbb{S}^{1}}\left(p_{2}, p_{2}^{\prime}\right)
$$

or other taxi products. 
The intrinsic flat $(\mathcal{F})$ distance is defined similarly to the Gromov-Hausdorff distance. Again we are taking an infimum over all distance preserving maps into a common metric space $Z$ which is now assumed to be complete instead of compact. Instead of taking the Hausdorff distance we measure the Federer-Fleming Flat Distance between the images. In full generality this flat distance is defined using Ambrosio-Kirchheim's mass measure of integral currents, $A$ and $B$, lying in $Z$ SW11. AK00.

Here we can take $Z=M \times I$ (where $I$ is an interval )with a well chosen metric $d_{Z}$ so that $\varphi_{i}: M_{i} \rightarrow Z$ are distance preserving maps such that

$$
\varphi_{0}(x)=(x, 0) \in Z \quad \forall x \in M_{0} \text { and } \varphi_{1}(x)=(x, 1) \in Z \quad \forall x \in M_{1} .
$$

Since we consider only $M$ without boundary we can set the filling current, $B$, to be integration over $M \times I$ and estimate its mass using the Hausdorff measure:

$$
d_{\mathcal{F}}\left(\left(M, d_{1}\right),\left(M, d_{0}\right)\right) \leq C_{m} \mathcal{H}_{d_{Z}}^{m+1}(M \times I) .
$$

This is an over estimate for the $\mathcal{F}$ distance but it suffices to show $\mathcal{F}$ convergence in this paper.

Note that in general both $\mathrm{GH}$ and $\mathcal{F}$ convergence are well defined for changing sequences of metric spaces and have compactness theorems defined in those settings. It is well known that even if one starts with a sequence of oriented Riemannian manifolds $M_{j}=\left(M, g_{j}\right)$ that the $\mathcal{F}$ and $\mathrm{GH}$ limits need not even be Riemannian manifolds. See [Gro81b] and [SW11] for these examples. It should also be noted that $\mathrm{GH}$ and $\mathcal{F}$ limits need not agree. There are many examples with no GH limit, that have $\mathcal{F}$ limits SW11.

If a sequence has a compact GH limit and the sequence has a uniform upper bound on volume, then a subsequence has a $\mathcal{F}$ limit and the $\mathcal{F}$ limit is either the zero space or a subset of the GH limit (See Theorem 3.20 in SW11]). Note that if $M_{j}$ has $\operatorname{Vol}_{j}\left(M_{j}\right) \rightarrow 0$ then $M_{j}$ converges in the intrinsic flat sense to the zero space. This can also happen without volume converging to 0 (See the Appendix to [SW11]). To prove that the limit is not the zero space, one can examine sequences of balls in $M_{j}$. By Lemma 4.1 of [Sor19] one sees that if $M_{j} \stackrel{\mathcal{F}}{\longrightarrow} M_{\infty}$ and $B\left(p_{j}, R\right) \in M_{j}$ endowed with the restricted metric $d_{j}$ converge to a limit $B_{\infty}(R)$, then for almost every $r \in(0, R), B_{\infty}(r)$ is isometric to a ball of radius $r$ in $M_{\infty}$. In particular if $B\left(p_{j}, R\right)$ do not converge to the zero space then $M_{\infty}$ is not the zero space either.

There are also theorems which provide hypotheses proving that $\mathcal{F}$ and GH limits exist and agree: see the work of Wenger, Matveev, Portegies, Perales, Nuñez-Zimbron, Huang, Lee, and the second author in [SW10] [PS17] MP17] NnZP17] [HLS17]. In the next subsection we describe the key result of Huang, Lee, and the second author that we apply to prove the main theorems in this paper. 
2.4. Useful Compactness Theorem. All the results in our paper apply the following compactness theorem to prove intrinsic flat $(\mathcal{F})$ and GromovHausdorff $(\mathrm{GH})$ convergence of our Riemannian manifolds. This theorem is an easy consequence of a theorem of Gromov in Gro81b and a theorem by Huang, Lee, and the third author in [HLS17]. Since both of those earlier theorems are stated in far greater generality than we need here, we simplify things by providing a direct proof here. Note that everything is much easier because we assume the spaces are Riemannian manifolds.

Theorem 2.3. If $M$ is a compact oriented Riemannian manifold with a sequence of continuous Riemannian metric tensors, $g_{j}$, and a background Riemannian metric, $g_{0}$, such that

$$
\lambda_{1} g_{0} \leq g_{j} \leq \lambda_{2} g_{0},
$$

then a subsequence converges in the uniform sense

$$
d_{j}: M \times M \rightarrow \mathbb{R} \quad \rightarrow \quad d_{\infty}: M \times M \rightarrow \mathbb{R}
$$

so that for some $K>0$

$$
d_{0}\left(q_{1}, q_{2}\right) \leq d_{\infty}\left(q_{1}, q_{2}\right) \leq K d_{0}\left(q_{1}, q_{2}\right) .
$$

In addition, for $M_{j}=\left(M, g_{j}\right)$ and $M_{\infty}=\left(M, d_{\infty}\right)$ we find

$$
\begin{aligned}
& M_{j} \stackrel{\mathcal{F}}{\longrightarrow} M_{\infty} \\
& M_{j} \stackrel{G H}{\longrightarrow} M_{\infty} .
\end{aligned}
$$

Note that in general uniform convergence of $d_{j} \rightarrow d_{\infty}$ does not imply $\mathcal{F}$ convergence due to the possibility of developing a cusp singularity. This can be seen in Example 3.4 of the first author's paper with Bryden on Sobolev bounds and the convergence of manifolds AB19.

Proof. First we recall that the Riemannian distances, $d_{j}$, defined by $g_{j}$ satisfy the following bi-Lipschitz bound for some $\lambda$ :

$$
\lambda \geq \frac{d_{j}(p, q)}{d_{0}(p, q)} \geq \frac{1}{\lambda}
$$

(cf. Lemma 2.2). Note immediately that there is now a uniform upper bound $D$ on the diameter of all the $M_{j}$,

$$
\operatorname{Diam}_{g_{j}}(M) \leq D=\lambda \operatorname{Diam}_{g_{0}}(M) .
$$

As in Gromov's argument in [Gro81b], applying the Arzela-Ascoli Theorem, a subsequence of the

$$
d_{j}: M \times M \rightarrow[0, D] \text { converges to } d_{\infty}: M \times M \rightarrow[0, D]
$$

and it can then be verified that $d_{\infty}$ is a length metric satisfying (41). So we have the claimed uniform convergence.

Gromov then explains in Gro81b] how this uniform convergence implies GH convergence. In fact Gromov-Hausdorff convergence is an extension of the notion of uniform convergence. We do not apply his proof. 
In the Appendix to [HLS17, Huang, Lee, and the second author prove both $\mathrm{GH}$ and $\mathcal{F}$ convergence by constructing a common metric space

$$
Z_{j}=\left[-\varepsilon_{j}, \varepsilon_{j}\right] \times M
$$

where

$$
\epsilon_{j}=\sup \left\{\left|d_{j}(p, q)-d_{\infty}(p, q)\right|: p, q \in M\right\}
$$

with a metric $d_{j}^{\prime}$ on $Z_{j}$ created by gluing together a pair of taxi products

$$
\left(Z_{j}^{-}, d_{j}^{\prime}\right)=\left[-\varepsilon_{j}, 0\right] \times_{\operatorname{taxi}}\left(M, d_{j}\right)
$$

and

$$
\left(Z_{j}^{+}, d_{j}^{\prime}\right)=\left[0, \varepsilon_{j}\right] \times_{t a x i}\left(M, d_{\infty}\right) .
$$

In the lemma in the Appendix of [HLS17] they prove there are distance preserving maps $\varphi_{j}:\left(M, d_{j}\right) \rightarrow\left(Z_{j}, d_{j}^{\prime}\right)$ and $\varphi_{j}^{\prime}:\left(M, d_{\infty}\right) \rightarrow\left(Z_{j}, d_{j}^{\prime}\right)$ such that

$$
\varphi_{j}(p)=\left(-\varepsilon_{j}, p\right) \text { and } \varphi_{j}^{\prime}(p)=\left(\varepsilon_{j}, p\right) .
$$

They also show

$$
d_{j}^{\prime}\left(z_{1}, z_{2}\right) \leq d_{0}^{\prime}\left(\left(t_{1}, p_{1}\right),\left(t_{2}, p_{2}\right)\right):=\left|t_{1}-t_{2}\right|+\lambda d_{0}\left(p_{1}, p_{2}\right) .
$$

Huang, Lee, and the third author then apply (49) to observe that every point

$$
\left(p,-\varepsilon_{j}\right)=\varphi_{j}(p) \in \varphi_{j}\left(M_{j}\right)
$$

has a point

$$
(p, \varepsilon)=\varphi_{j}^{\prime}(p) \in \varphi_{j}^{\prime}\left(M_{\infty}\right)
$$

such that

$$
d_{j}^{\prime}\left(\left(p,-\varepsilon_{j}\right),(p, \varepsilon)\right)=2 \varepsilon_{j}
$$

and visa versa. Thus

$$
d_{G H}\left(M_{j}, M_{\infty}\right) \leq d_{H}^{Z_{j}}\left(\varphi_{j}\left(M_{j}\right), \varphi_{j}^{\prime}\left(M_{\infty}\right)\right)=2 \varepsilon_{j} \rightarrow 0 .
$$

To estimate the intrinsic flat distance one then needs only estimate

$$
\begin{aligned}
d_{\mathcal{F}}\left(M_{j}, M_{\infty}\right) & \leq C_{m} \mathcal{H}_{d_{0}^{\prime}}^{m+1}\left(Z_{j}\right) \\
& \leq C_{m} 2 \varepsilon_{j} \lambda^{m} \mathcal{H}_{d_{0}}^{m}(M)
\end{aligned}
$$

More details on this with more precise constants appear in the Appendix to HLS17. 


\subsection{Metric Measure Convergence and Volume Preserving Intrinsic}

Flat Convergence. The notion of metric measure convergence first introduced by Fukaya in [Fuk87, and studied by Cheeger-Colding in [CC97, and generalized by Sturm to an intrinsic Wasserstein distance in Stu06a, Stu06b is defined on a large class of spaces as well. If one has a sequence of compact oriented Riemannian manifolds converging in the GH sense such that whenever points $p_{j} \in M_{j}$ converge to $p_{\infty} \in M_{\infty}$ the volumes of the balls around them converge to the measure of the limit ball,

$$
\operatorname{Vol}\left(B\left(p_{j}, r\right)\right) \rightarrow \mu\left(B\left(p_{\infty}, r\right)\right)
$$

then one has metric measure convergence in all these respects to $\left(M_{\infty}, d_{\infty}, \mu\right)$. One might ask how $p_{j} \in M_{j}$ converge to $p_{\infty} \in M_{\infty}$ when they do not lie in a common space. This is said to hold when there are distance preserving maps $\varphi_{j}: M_{j} \rightarrow Z$ and $\varphi_{\infty}: M_{\infty} \rightarrow Z$ such that

$$
d_{H}^{Z}\left(\varphi_{j}\left(M_{j}\right), \varphi_{\infty}\left(M_{\infty}\right)\right) \rightarrow 0 \text { and } \varphi_{j}\left(p_{j}\right) \rightarrow \varphi_{\infty}\left(p_{\infty}\right) .
$$

The existence of such a common compact $Z$ was proven in general by Gromov in Gro81a whenever a sequence converges in the GH sense to a compact limit. The existence of a common complete $Z$ was proven in [SW11] for intrinsic flat converging sequences and studied further in Sor19.

A common $Z$ that works for both $\mathcal{F}$ and GH convergence in the setting of Theorem 2.3 was constructed by the authors in the Appendix to [AB19] by attaching the many $Z_{j}$ like pages along the $M_{\infty}$ edge. There we saw that if we have $\left(M, g_{j}\right)$ and take $p_{j}=p$ a fixed point in $M$ viewed in each $M_{j}$ then $p_{\infty}=p$ as well now viewed in $M_{\infty}$. Thus one can simplify the definition in our setting to say that $M_{j}=\left(M, g_{j}\right)$ converges in the metric measure sense to $\left(M, d_{\infty}\right)$ if it converges in the Gromov-Hausdorff sense and

$$
\operatorname{Vol}_{j}(B(p, r)) \rightarrow \mathcal{H}_{d_{\infty}}^{m}(B(p, r)) \quad \forall p \in M \quad \forall r>0 .
$$

Volume preserving intrinsic flat $(\mathcal{V F})$ convergence is defined

$$
M_{j} \stackrel{\mathcal{V F}}{\longrightarrow} M_{\infty} \Longleftrightarrow M_{j} \stackrel{\mathcal{F}}{\longrightarrow} M_{\infty} \operatorname{AND}_{\operatorname{Vol}_{g_{j}}}\left(M_{j}\right) \rightarrow \operatorname{Vol}_{g_{\infty}}\left(M_{\infty}\right) .
$$

when the sequence and the limit are both Riemannian manifolds. This has been studied by Jauregui and Lee in [JL19. It should be noted that the second author and Wenger proved in [SW11] (see also [Sor19] PS17]) that if $M_{j} \stackrel{\mathcal{F}}{\longrightarrow} M_{\infty}$ and $p_{j} \rightarrow p_{\infty}$ then for almost every $r>0 B\left(p_{j}, r\right) \stackrel{\mathcal{F}}{\longrightarrow} B\left(p_{\infty}, r\right)$ and

$$
\liminf _{j \rightarrow \infty} \operatorname{Vol}_{g_{j}}\left(B\left(p_{j}, r\right)\right) \geq \operatorname{Vol}_{g_{\infty}}\left(B\left(p_{\infty}, r\right)\right) .
$$

If one requires $\operatorname{Vol}_{g_{j}}\left(M_{j}\right) \rightarrow \operatorname{Vol}_{g_{\infty}}\left(M_{\infty}\right)$ then no balls can drop in volume in the limit. Thus

$$
\left(M_{j}, g_{j}\right) \stackrel{\mathcal{V F}}{\longrightarrow}\left(M_{\infty}, g_{\infty}\right) \Longrightarrow \operatorname{Vol}_{g_{j}}\left(B\left(p_{j}, r\right)\right) \rightarrow \operatorname{Vol}_{g_{\infty}}\left(B\left(p_{\infty}, r\right)\right) .
$$

In particular if we also have $\left(M_{j}, g_{j}\right) \stackrel{\mathrm{GH}}{\longrightarrow}\left(M_{\infty}, g_{\infty}\right)$ then we have $\left(M_{j}, g_{j}\right) \stackrel{\mathrm{mGH}}{\longrightarrow}$ $\left(M_{\infty}, g_{\infty}\right)$. 
We summarize this in a theorem which should be viewed as a simplification of known theorems:

Theorem 2.4. Let $M$ be a compact oriented manifold and $M_{j}=\left(M, g_{j}\right)$ and $M_{\infty}=\left(M, g_{\infty}\right)$ be Riemannian manifolds. If $M_{j} \stackrel{G H}{\longrightarrow} M_{\infty}, M_{j} \stackrel{\mathcal{F}}{\longrightarrow} M_{\infty}$, and

$$
\operatorname{Vol}_{g_{j}}\left(M_{j}\right) \rightarrow \operatorname{Vol}_{g_{\infty}}\left(M_{\infty}\right)
$$

then $M_{j} \stackrel{\mathcal{V F}}{\longrightarrow} M_{\infty}$ and $M_{j} \stackrel{m G H}{\longrightarrow} M_{\infty}$.

2.6. Lebesgue Convergence of Riemannian Manifolds. Above we have reviewed four geometric notions of convergence that are all weaker than $C^{0}$ convergence of metric tensors. However geometric analysts will find it more natural to weaken the notion of $C^{0}$ convergence to $L^{p}$ convergence of metric tensors. Indeed when studying sequences of Riemannian manifolds it can be easier for geometric analysts to prove $L^{p}$ convergence rather than $\mathrm{GH}$ or $\mathcal{F}$ convergence. Our results here can be thought of as analogous to how in PDEs one will start by obtaining $L^{p}$ bounds in order to use this control to then bootstrap up to stronger control in the future.

Here we review the definition of $L^{p}$ norm for Riemannian metrics and discuss important properties for this paper. See the work of Clarke Cla11] for these definitions used for a study of the space of Riemannian metrics on a fixed background manifold with respect to the $L^{2}$ topology.

If we consider the compact manifold $M$ and the Riemannian metrics $\left(M, g_{0}\right)$ and $\left(M, g_{1}\right)$ then we can define the $L^{p}, p \geq 1$ norm of $g_{1}$ with respect to the background metric $g_{0}$ to be

$$
\left\|g_{1}\right\|_{L_{g_{0}}^{p}(M)}=\left(\int_{M}\left|g_{1}\right|_{g_{0}}^{p} d V_{g_{0}}\right)^{1 / p} .
$$

Notice that this is just the usual definition of the $L^{p}$ norm on $\left(M, g_{0}\right)$ for the function $\left|g_{1}\right|_{g_{0}}$ which is defined by letting $\lambda_{1}^{2}, \ldots, \lambda_{m}^{2}$ be the eigenvalues of $g_{1}$ with respect to $g_{0}$ with corresponding eigenvectors $v_{1}, \ldots, v_{m}$ so that

$$
g_{1}\left(v_{i}, v_{i}\right)=\lambda_{i}^{2} g_{0}\left(v_{i}, v_{i}\right)=\lambda_{i}^{2}, \quad 1 \leq i \leq m,
$$

and

$$
\left|g_{1}\right|_{g_{0}}=\sqrt{\sum_{i=1}^{m} \lambda_{i}^{4}}
$$

the norm of $g_{1}$ with respect to $g_{0}$. In fact, if $g_{1}=f_{1}^{2} g_{0}$ is a conformal metric then

$$
\left\|g_{1}\right\|_{L_{g_{0}}^{\frac{p}{2}}(M)}=m^{\frac{1}{2}}\left\|f_{1}\right\|_{L_{g_{0}}^{p}(M)}^{2}=m^{\frac{1}{2}}\left(\int_{M}\left|f_{1}\right|^{p} d V_{g_{0}}\right)^{2 / p}
$$


Hence we say that a sequence of Riemannian manifolds $\left(M, g_{j}\right)$ converges to the Riemannian manifold $\left(M, g_{\infty}\right)$ in $L^{p}$ norm with respect to $g_{0}$ if

$$
\left\|g_{j}-g_{\infty}\right\|_{L_{g_{0}}^{p}(M)} \rightarrow 0 .
$$

The following lemma follows from Hölder's Inequality:

Lemma 2.5. Let $g_{j}, g_{0}$ be continuous Riemannian metrics defined on the compact manifold $M$. For $p \in(1, \infty]$ if

$$
\left\|g_{j}-g_{0}\right\|_{L_{g_{0}}^{p}(M)} \rightarrow 0
$$

then

$$
\left\|g_{j}-g_{0}\right\|_{L_{g_{0}}^{q}(M)} \rightarrow 0, \quad 1 \leq q \leq p .
$$

In general the careful treatment of the power of $p$ in $L^{p}$ convergence is crucial. However when we assume a uniform upper bound on the metric tensors as in our main theorem, the specific value of $p$ chosen in $L^{p}$ convergence is not particularly important:

Lemma 2.6. If $\left(M, g_{j}\right),\left(M, g_{0}\right)$ are compact continuous Riemannian manifolds so that

$$
g_{j}(v, v) \leq K g_{0}(v, v), \quad \forall v \in T_{p} M
$$

and for $p \in[1, \infty]$

$$
\left\|g_{j}-g_{0}\right\|_{L_{g_{0}}^{p}(M)} \rightarrow 0
$$

then

$$
\left\|g_{j}-g_{0}\right\|_{L_{g_{0}}^{q}(M)}, \forall q \in[1, \infty] .
$$

Proof. We know by Hölder's inequality that

$$
\left\|g_{j}-g_{0}\right\|_{L_{g_{0}}^{q}(M)} \rightarrow 0, \quad 1 \leq q \leq p .
$$

Otherwise, we calculate for $q>p$

$$
\begin{aligned}
\left\|g_{j}-g_{0}\right\|_{L_{g_{0}}^{q}(M)}^{q} & =\int_{M}\left|g_{j}-g_{0}\right|^{q} d V_{g_{0}} \\
& =\int_{M}\left|g_{j}-g_{0}\right|^{p}\left|g_{j}-g_{0}\right|^{q-p} d V_{g_{0}} \\
& \leq(2 K \sqrt{m})^{q-p} \int_{M}\left|g_{j}-g_{0}\right|^{p} d V_{g_{0}} \rightarrow 0 .
\end{aligned}
$$

We now state a standard analysis result relating convergence in $L^{p}$ to convergence in $L^{p}$ norm. 
Lemma 2.7. Assume that $M_{j}^{m}=\left(M, g_{j}\right), M_{\infty}=\left(M, g_{\infty}\right)$, and $M_{0}=$ $\left(M, g_{0}\right)$ are continuous Riemannian manifolds. If for $p \in[1, \infty)$

$$
\int_{M}\left|g_{j}-g_{\infty}\right|_{g_{0}}^{p} d V_{g_{0}} \rightarrow 0
$$

then

$$
\left\|g_{j}\right\|_{L_{g_{0}}^{p}(M)} \rightarrow\left\|g_{\infty}\right\|_{L_{g_{0}}^{p}(M)}
$$

Proof. By the reverse triangle inequality applied to norms

$$
\left|\left\|g_{j}\right\|_{L_{g_{0}}^{p}(M)}-\left\|g_{\infty}\right\|_{L_{g_{0}}^{p}(M)}\right| \leq\left\|g_{j}-g_{\infty}\right\|_{L_{g_{0}}^{p}(M)},
$$

we find that $g_{j}$ converging to $g_{\infty}$ in $L_{g_{0}}^{p}(M)$ implies convergence in $L_{g_{0}}^{p}(M)$ norm.

In a previous paper by the authors [AS19] a comprehensive comparison of $L^{p}$ convergence and the uniform, $\mathrm{GH}$, and $\mathcal{F}$ convergence of warped products Riemannian manifolds was given. These are Riemannian manifolds whose metric tensor has the form $g=d t^{2}+f^{2}(t) g_{0}$. The authors gave a theorem which assumed metric bounds and $L^{2}$ convergence of warping factors, $f_{j} \rightarrow$ $f_{0}$ which implied uniform, GH, and $\mathcal{F}$ convergence to a warped product with warping function $f_{0}$. That theorem is now a special case of the the Theorem 1.2 proven here.

The authors also produced many examples contrasting different notions of convergence in [AS19. In particular they constructed an examples which show that $L^{p}$ convergence of Riemannian manifolds need not agree with geometric notions of convergence like $\mathrm{GH}$ and $\mathcal{F}$ convergence. This is due to the fact that the $L^{p}$ norm considers $g_{j}$ and $g_{0}$ to be close even if they measure the lengths of vectors very differently on a set of measure zero $S \subset M$. In the examples where the notions of convergence disagree, the set $S$ contains geodesics with respect to $g_{\infty}$. It is particularly a concern if the $g_{j}$ are much smaller than $g_{0}$ on the set $S$ providing shortcuts for the $d_{j}$ so that the limit of the $d_{j}$ ends up smaller than $d_{\infty}$. There are pictures in AS19 illustrating exactly what is happening. To avoid this trouble the hypothesis that $g_{j} \geq(1-1 / j) g_{0}$ was first introduced in [AS19] rather than simply $g_{j} \geq\left(\lambda_{1}\right) g_{0}$ as is needed to apply the useful compactness theorem (cf. Theorem 2.3). Example 3.1 within this paper demonstrates that the same issue arises when studying conformal sequences of manifolds and so we also require this hypothesis in our new Theorem 1.2 ,

\section{Examples of Sequences of Conformal Manifolds}

In this section we explore sequences of metrics on a torus that are conformal to the flat torus and metrics on a sphere that are conformal to the standard sphere. The first three examples are all uniformly bounded and directly explore hypotheses and conclusions related to Theorem 1.1. The 
last five examples explore what can happen when the uniform upper bound of Theorem 1.1 is removed.

We also direct the reader to the warped product examples given in our previous paper [AS19] which are relevant to Theorem 1.2.

3.1. Without Lower Bounds on the Conformal Factor. Here we see an example which shows that without the $C^{0}$ bound from below one cannot prove convergence of $M_{j}$ to $M_{0}$ in Theorem 1.1. Note examples demonstrating why the lower bounds are needed on the metric tensor itself as in Theorem 1.2 appeared in the authors' previous paper on warped products AS19.

Example 3.1. Consider the sequence of functions on $\mathbb{S}^{m}$ which are radially defined from the north pole

$$
f_{j}(r)= \begin{cases}1 & r \in[0, \pi / 2-1 / j] \\ h(j r-\pi / 2) & r \in[\pi / 2-1 / j, \pi / 2+1 / j] \\ 1 & r \in[\pi / 2+1 / j, \pi]\end{cases}
$$

where $h:[-1,1] \rightarrow \mathbb{R}$ is a smooth even function such that $h(-1)=1$ with $h^{\prime}(-1)=0$, decreasing to $h(0)=h_{0} \in(0,1)$ and then increasing back up to $h(1)=1, h^{\prime}(1)=0$. We will see that for $M_{j}=\left(\mathbb{S}^{m}, f_{j}^{2} g_{\mathbb{S}^{m}}\right)$

$$
\begin{aligned}
& M_{j} \stackrel{\mathcal{V F}}{\longrightarrow} M_{\infty} \\
& M_{j} \stackrel{m G H}{\longrightarrow} M_{\infty}
\end{aligned}
$$

but we can conclude that $M_{\infty}$ is not isometric to $\mathbb{S}^{m}$. Instead $M_{\infty}=$ $\left(\mathbb{S}^{m}, f_{\infty}^{2} g_{\mathbb{S}^{m}}\right)$ is the conformal metric with conformal factor

$$
f_{\infty}(r)=\left\{\begin{array}{lc}
h_{0} & r=\pi / 2 \\
1 & \text { otherwise }
\end{array} .\right.
$$

The distances between pairs of points near the equator in this limit space is achieved by geodesics which run to the equator, and then around inside the cinched equator, and then out again.

Proof. Notice that $h_{0} \leq f_{j} \leq 1$ and hence by Theorem 2.3 we find that on a subsequence

$$
\begin{aligned}
& M_{j} \stackrel{\mathcal{F}}{\longrightarrow} M^{\prime}, \\
& M_{j} \stackrel{\mathrm{GH}}{\longrightarrow} M^{\prime},
\end{aligned}
$$

for some compact metric space $M^{\prime}=\left(\mathbb{S}^{m}, d^{\prime}\right)$ where we have uniform convergence $d_{j} \rightarrow d^{\prime}$. Now our goal is to show that $M^{\prime}=M_{\infty}$ by showing pointwise convergence $d_{j}\left(q_{1}, q_{2}\right) \rightarrow d_{\infty}\left(q_{1}, q_{2}\right)$ for all $q_{1}, q_{2} \in \mathbb{S}^{m}$. Thus $d^{\prime}=d_{\infty}$. 
Let $q_{1}, q_{2} \in \mathbb{S}^{m}$ and consider $\gamma(t)=(r(t), \theta(t))$ to be any curve in $\mathbb{S}^{m}$. We can compute

$$
d_{j}\left(q_{1}, q_{2}\right) \leq L_{j}(\gamma)=\int_{0}^{L} f_{j}(r(t)) \sqrt{r^{\prime}(t)^{2}+\theta^{\prime}(t)^{2}} d t \rightarrow L_{\infty}(\gamma)
$$

where the convergence follows from the dominated convergence theorem since $f_{j}(r(t)) \rightarrow f_{\infty}(r(t))$ pointwise and $f_{j}$ is uniformly bounded. Since this is true for any curve $\gamma$ we see that

$$
\limsup _{j \rightarrow \infty} d_{j}\left(q_{1}, q_{2}\right) \leq d_{\infty}\left(q_{1}, q_{2}\right)
$$

Now let $\gamma_{j}(t)=\left(r_{j}(t), \theta_{j}(t)\right)$ be the length minimizing curve with respect to $g_{j}$ defined on $t \in[0, L]$. Then if we define

$$
\begin{gathered}
S_{j}=\left\{t \in[0, L]: \pi / 2-1 / j \leq r_{j}(t) \leq \pi / 2+1 / j, r_{j}(t) \neq \frac{\pi}{2}\right\}, \\
T_{j}=\left\{t \in[0, L]: r_{j}(t)=\frac{\pi}{2}\right\},
\end{gathered}
$$

and

$$
U_{j}=[0,1] \backslash\left(S_{j} \cup T_{j}\right)
$$

we can compute

$$
\begin{aligned}
d_{j}\left(q_{1}, q_{1}\right) & =L_{j}\left(\gamma_{j}\right) \\
& =L_{j}\left(U_{j}\right)+L_{j}\left(T_{j}\right)+L_{j}\left(S_{j}\right) \\
& =L_{\infty}\left(U_{j}\right)+h_{0} L_{\mathbb{S}^{m}}\left(T_{j}\right)+L_{j}\left(S_{j}\right) \\
& \geq d_{\infty}\left(q_{1}, q_{2}\right)+L_{j}\left(S_{j}\right)-L_{\mathbb{S}^{m}}\left(S_{j}\right) .
\end{aligned}
$$

Now we notice

$$
\left|L_{j}\left(S_{j}\right)-L_{\mathbb{S} m}\left(S_{j}\right)\right| \leq \int_{0}^{L_{\mathbb{S} m}\left(S_{j}\right)}\left|h_{j}\left(r_{j}(t), \theta_{j}(t)\right)-1\right| d t \rightarrow 0
$$

since $L_{\mathbb{S} m}\left(S_{j}\right) \leq L<\infty$, otherwise we would contradict (87), and the dominated convergence theorem. Hence we find

$$
\liminf _{j \rightarrow \infty} d_{j}\left(q_{1}, q_{2}\right) \geq d_{\infty}\left(q_{1}, q_{2}\right) .
$$

By combining (87) and (96) we find pointwise convergence of distances which implies that $\left(M^{\prime}, d^{\prime}\right)=\left(M_{\infty}, d_{\infty}\right)$. It is also clear that the volume converges in this example.

3.2. Conformal Tori converging as in Theorem 1.1 to a Flat Torus. Here we give an example which fits the hypotheses of the Theorem 1.2 and comes to the same conclusion but whose conformal factors do not converge in $C^{0}$. This shows that one should not expect to conclude any stronger convergence in Theorem 1.2. 
Example 3.2. Consider the sequence of functions on $\mathbb{T}^{m}$ which are radially defined from a point $p \in \mathbb{T}^{m}$

$$
f_{j}(r)= \begin{cases}K & \text { if } r \in[0,1 / j] \\ h_{j}(j r) & \text { if } r \in[1 / j, 2 / j] \\ 1 & \text { if } r \in(1 / j, \sqrt{2} \pi]\end{cases}
$$

where $K \in(1, \infty)$ and $h_{j}:[1,2] \rightarrow \mathbb{R}$ is a smooth, decreasing function so that $h_{j}(1)=K$ and $h_{j}(2)=1$. Then $M_{j}=\left(\mathbb{T}^{m}, f_{j}^{2} g_{\mathbb{T}^{m}}\right)$ converges to $\mathbb{T}^{m}$ in $L^{p} \forall p \in(0, \infty)$, but does not converge to $\mathbb{T}^{m}$ in $C^{0}$, and rather

$$
\begin{aligned}
& M_{j} \stackrel{\mathcal{V \mathcal { F }}}{\longrightarrow} \mathbb{T}^{m} \\
& M_{j} \stackrel{G H}{\longrightarrow} \mathbb{T}^{m} .
\end{aligned}
$$

Proof. We begin by computing

$$
\begin{aligned}
\operatorname{Vol}\left(M_{j}\right)= & \int_{\mathbb{T}^{m}} f_{j}^{m} d V o l \\
= & \operatorname{Vol}\left(B^{m}(p, 1 / j)\right) K \\
& +\omega_{m} \int_{1 / j}^{2 / j} h_{j}(j r)^{m} r^{m-1} d r \\
& +\operatorname{Vol}\left(\mathbb{T}^{m} \backslash B^{m}(p, 2 / j) \rightarrow \operatorname{Vol}\left(\mathbb{T}^{m}\right),\right. \\
\operatorname{Diam}\left(M_{j}\right)= & \int_{0}^{\sqrt{2} \pi} f_{j} d r \\
= & \frac{K}{j}+\int_{1 / j}^{2 / j} h_{j}(j r) d r+(\sqrt{2} \pi-1 / j) \rightarrow \sqrt{2} \pi,
\end{aligned}
$$

and

$$
\left\|f_{j}-1\right\|_{L^{p}\left(\mathbb{T}^{m}\right)} \leq \operatorname{Vol}(B(p, 2 / j))(K-1)^{p} \rightarrow 0 .
$$

Hence $M_{j} \stackrel{\mathrm{mGH}}{\longrightarrow} \mathbb{T}^{m}$ and $M_{j} \stackrel{\mathcal{V F}}{\longrightarrow} \mathbb{T}^{m}$ by Theorem 1.1 but we note that $f_{j}$ clearly does not converge uniformly to 1

3.3. Only Pointwise Convergence of the Conformal Factor. Here we give an example of a sequence of conformally flat tori whose conformal factors pointwise converge on a dense set to 1 , but do not converge in $L^{p}$ to 1 . The conformal factors do satisfy a lower bound $f_{j} \geq 1$ and a uniform upper bound, but $M_{j}$ do not GH nor $\mathcal{F}$ converge to $M_{0}$. This shows that the assumption of $L^{p}$ convergence or volume convergence in Theorem 1.2 cannot be weakened to pointwise convergence on a dense set.

Example 3.3. Define the $j$ th lattice

$$
L_{j}=\left\{\left(\frac{n}{2^{j}}, r\right) \cup\left(r, \frac{m}{2^{j}}\right): r \in[0,1], n, m=1 \ldots 2^{j}\right\},
$$


and the limiting set

$$
L=\bigcup_{j=1}^{\infty} L_{j} .
$$

Consider $M_{j}=\left(\mathbb{T}^{m}, g_{j}=f_{j}^{2} g_{0}\right)$ with conformal factor,

$$
f_{j}=\left\{\begin{array}{ll}
1 & \text { on } T_{\frac{1}{2^{j+2}}}\left(L_{j}\right) \\
\sqrt{2} & \text { elsewhere }
\end{array},\right.
$$

where $T_{r}(S)$ represents a tubular neighborhood of radius $r>0$ around the set $S \subset \mathbb{T}^{m}$. Then

$$
f_{j}(p) \rightarrow 1, \quad p \in L
$$

pointwise on the dense set $L$,

$$
f_{j}(p) \rightarrow \sqrt{2}, \quad p \in \mathbb{T}^{m} \backslash L
$$

pointwise on the full measure set set $\mathbb{T}^{m} \backslash L$, and

$$
g_{j} \geq g_{\mathbb{T}^{m}},
$$

but yet $M_{j}$ does not converge to $\mathbb{T}^{m}$. Instead

$$
\begin{aligned}
& M_{j} \stackrel{G H}{\longrightarrow} \mathbb{T}_{\text {taxi }}^{m}, \\
& M_{j} \stackrel{\mathcal{F}}{\longrightarrow} \mathbb{T}_{\text {taxi }}^{m},
\end{aligned}
$$

where $\mathbb{T}_{\text {taxi }}^{m}=\left(\mathbb{T}^{m}, d_{\text {taxi }}\right)$ is a torus with a taxi metric.

Proof. First we notice that for any $p, q \in M_{j}$ the length minimizing geodesic from $p$ to $q$ consists of a collection of straight line segments. Furthermore, no segment should enter a region where $f_{j}=\sqrt{2}$, unless it begins or ends in one, since

$$
\sqrt{2} \sqrt{x^{2}+y^{2}} \geq|x|+|y| .
$$

By this observation we notice that for all $p \in M_{j}$ there exists $\left(\frac{n}{2^{j}}, \frac{m}{2^{j}}\right)$ so that

$$
d_{j}\left(p,\left(\frac{n}{2^{j}}, \frac{m}{2^{j}}\right)\right) \leq \sqrt{2}\left(\frac{\sqrt{2}}{2^{j}}\right),
$$

which is an overestimate of the distance from the center of the square to one of its corners. So (116) shows that $L_{j}$ is a $\frac{1}{2^{j-1}}$ net and since by (115)

$$
d_{j}(p, q) \rightarrow d_{\text {taxi }}(p, q) \quad \forall p, q \in L
$$

we have that

$$
M_{j} \stackrel{\mathrm{GH}}{\longrightarrow} \mathbb{T}_{\text {taxi }}^{m} .
$$

Then since

$$
g_{0} \leq g_{j} \leq \sqrt{2} g_{0}
$$


we have by Theorem 2.3 that the $\mathcal{F}$ limit agrees with the GH limit.

3.4. Conformal Tori converging in $L^{p}, p<\frac{m}{\alpha}, \alpha \in(0,1)$. Here we see an example which satisfies $g_{j} \geq g_{0}$ and whose conformal factor $f_{j}$ converges in $L^{p}$ norm for all $p<\frac{m}{\alpha}, \alpha \in(0,1)$ which is a stronger assumption than volume convergence, and hence we see that $M_{j}$ converges in both the $\mathrm{mGH}$ and $\mathcal{V} \mathcal{F}$ sense to a flat torus.

Example 3.4. Consider the sequence of functions on $\mathbb{T}^{m}$ which are radially defined from a point $p \in \mathbb{T}^{m}$

$$
f_{j}(r)= \begin{cases}j^{\alpha} & \text { if } r \in[0,1 / j] \\ h_{j}(j r) & \text { if } r \in[1 / j, 2 / j] \\ 1 & \text { if } r \in(1 / j, \sqrt{2} \pi]\end{cases}
$$

where $0<\alpha<1$ and $h_{j}:[1,2] \rightarrow \mathbb{R}$ is a smooth, decreasing function so that $h_{j}(1)=j^{\alpha}$ and $h_{j}(2)=1$. Then for $M_{j}=\left(\mathbb{T}^{m}, f_{j}^{2} g_{\mathbb{T}^{m}}\right)$

$$
\begin{gathered}
\left\|f_{j}-1\right\|_{L^{p}\left(\mathbb{T}^{m}\right)} \rightarrow 0 \text { for } p<\frac{m}{\alpha}, \\
1 \leq f_{j}
\end{gathered}
$$

and

$$
\begin{aligned}
& M_{j} \stackrel{\mathcal{V F}}{\longrightarrow} \mathbb{T}^{m} \\
& M_{j} \stackrel{m G H}{\longrightarrow} \mathbb{T}^{m} .
\end{aligned}
$$

Proof. We begin by computing

$$
\begin{aligned}
\operatorname{Vol}\left(M_{j}\right)= & \int_{\mathbb{T}^{m}} f_{j}^{m} d V o l \\
\leq & \operatorname{Vol}\left(B^{m}(p, 2 / j)\right) j^{m \alpha} \\
& +\omega_{m} \int_{1 / j}^{2 / j} h_{j}(j r)^{m} r^{m-1} d r \\
& \quad+\operatorname{Vol}\left(\mathbb{T}^{m} \backslash B^{m}(p, 2 / j) \rightarrow \operatorname{Vol}\left(\mathbb{T}^{m}\right),\right. \\
\operatorname{Diam}\left(M_{j}\right)= & \int_{0}^{\sqrt{2} \pi} f_{j} d r \\
= & j^{\alpha-1}+\int_{1 / j}^{2 / j} h_{j}(j r) d r+(\sqrt{2} \pi-1 / j) \rightarrow \sqrt{2} \pi,
\end{aligned}
$$

and

$$
\begin{aligned}
\left\|f_{j}-1\right\|_{L^{p}\left(\mathbb{T}^{m}\right)} & \leq \operatorname{Vol}(B(p, 2 / j))\left(j^{\alpha}-1\right)^{p} \\
& =2^{m} \omega_{m} \frac{\left(j^{\alpha}-1\right)^{p}}{j^{m}} \\
& \leq 2^{m} \omega_{m} j^{\alpha p-m} \rightarrow 0, \quad p<\frac{m}{\alpha} .
\end{aligned}
$$


Now by Lemma 2.2 since $f_{j} \geq 1$ we know

$$
d_{j}\left(q_{1}, q_{2}\right) \geq d_{0}\left(q_{1}, q_{2}\right) \quad \forall q_{1}, q_{2} \in \mathbb{T}^{m} .
$$

For $q_{1}, q_{2} \in \mathbb{T}^{m}$ so that the $g_{0}$ length minimizing geodesic, $\gamma$, does not pass through $p$ we notice that for $j$ chosen large enough $L_{j}(\gamma)=L_{0}(\gamma)$ and hence

$$
d_{j}\left(q_{1}, q_{2}\right) \leq L_{j}(\gamma)=L_{0}(\gamma)=d_{0}(p, q) .
$$

If $\gamma$ does pass through $p$ then it is enough to consider $p, q \in \mathbb{T}^{m}$ and for $j$ chosen large enough we have that $q \notin B(p, 2 / j)$ and hence we can calculate

$$
d_{j}(p, q) \leq \int_{0}^{d_{0}(p, q)} f_{j} d r=2 j^{\alpha-1}+d_{0}(p, q)-\frac{2}{j}=d_{0}(p, q)+2 \frac{j^{\alpha}-1}{j} .
$$

Now by combining (134), (135), and (136) we find $d_{j} \rightarrow d_{0}$ uniformly and hence

$$
M_{j} \stackrel{\mathrm{mGH}}{\longrightarrow} \mathbb{T}^{m} .
$$

Once $M_{j}$ has a GH limit $M_{\infty}$ then by Theorem 3.20, p. 147 of [SW11] we know that a subsequence converges in the $\mathcal{F}$ sense either to the 0 space or a subset of the GH limit. Since the $\mathcal{F}$ limit must have the same dimension as the sequence, the subsequence converges in the $\mathcal{F}$ sense either to the 0 space or a subset of the flat torus. Now consider any point $q \neq p$ in the flat torus and consider $B_{d_{j}}(q, r) \in M_{j}$ where $r<d_{0}(p, q) \leq d_{j}(p, q)$. For $j$ sufficiently large this is a Euclidean ball, and so by Lemma 4.1 in [Sor19], choosing possibly a smaller $r>0, B_{d_{j}}(q, r) \in M_{j}$ converges to a Euclidean ball of radius $r$ inside the $\mathcal{F}$ limit, $M_{\infty}$. Piecing together these Euclidean balls, we see that the intrinsic flat limit is a flat torus with possibly one point removed. But that point is added back in when one takes the metric completion and hence we find $\mathcal{V F}$ convergence. A new proof of this limit will appear in upcoming work of the authors.

3.5. Conformal Tori which don't converge in $L^{m}$. Here we see an example of a sequence of metrics on tori whose conformal factor $f_{j}$ is not bounded in $L^{p}$ for any $p>m$, is bounded in $L^{m}$ but does not converge in $L^{m}$, and does not satisfy volume convergence. This example will converge in both the GH and $\mathcal{F}$ sense to a metric space which is isometric to a flat torus with a flat disk attached so that the boundary of the disk is identified with a point in the flat torus. This shows that volume convergence is important in order to prevent bubbling. From an analytic point of view this sequence of conformal factors are converging to a measure whose mass is concentrating at a point which geometrically corresponds to a flat torus with a bubble attached at a point. 
Example 3.5. Consider the sequence of functions on $\mathbb{T}^{m}$ which are radially defined from a point $p \in \mathbb{T}^{m}$

$$
f_{j}(r)= \begin{cases}j & \text { if } r \in[0,1 / j] \\ h_{j}(j r) & \text { if } r \in[1 / j, 2 / j] \\ 1 & \text { if } r \in(2 / j, \sqrt{m} \pi] .\end{cases}
$$

where $h_{j}:[1,2] \rightarrow \mathbb{R}$ is a smooth, decreasing function so that $h_{j}(1)=j$, $h_{j}^{\prime}(1)=h_{j}^{\prime}(2)=0$, and $h_{j}(2)=1$ so that

$$
\frac{1}{j^{m}} \int_{1}^{2} h_{j}(s)^{m} s^{m-1} d s \rightarrow 0 .
$$

Then $f_{j}$ is not bounded in $L^{p}$ norm for $p>m$ but does have bounded $L^{m}$ norm and volume. Furthermore, for $M_{j}=\left(\mathbb{T}^{m}, f_{j}^{2} g_{\mathbb{T}^{m}}\right)$

$$
\begin{aligned}
& M_{j} \stackrel{\mathcal{F}}{\longrightarrow} M_{\infty} \\
& M_{j} \stackrel{G H}{\longrightarrow} M_{\infty}
\end{aligned}
$$

where $M_{\infty}$ is not isometric to $\mathbb{T}^{m}$. Instead

$$
M_{\infty}=\mathbb{T}^{m} \sqcup \mathbb{D}^{m} / \sim,
$$

where we fix a $p \in \mathbb{T}^{m}$ and for $d \in \partial \mathbb{D}^{m}$ we have

$$
p \sim d
$$

Proof. First notice by Holder's inequality that the assumption (139) implies

$$
\begin{aligned}
\frac{1}{j} \int_{1}^{2} h_{j}(r) d r & \leq \frac{1}{2 j} \int_{1}^{2} h_{j}(r) r^{\frac{m-1}{m}} d r \\
& \leq \frac{1}{2 j}\left(\int_{1}^{2} h_{j}(r)^{m} r^{m-1} d r\right)^{1 / m} \\
& =\frac{1}{2}\left(\frac{1}{j^{m}} \int_{1}^{2} h_{j}(r)^{m} r^{m-1} d r\right)^{1 / m} \rightarrow 0 .
\end{aligned}
$$

Now we begin by computing

$$
\begin{aligned}
& \operatorname{Vol}\left(M_{j}\right)=\int_{\mathbb{T}^{m}} f_{j}^{m} d \operatorname{Vol} \\
& =\operatorname{Vol}\left(B^{m}(p, 1 / j)\right) j^{m}+\operatorname{Vol}\left(\mathbb{T}^{m} \backslash B^{m}(p, 2 / j)\right) \\
& \quad \quad+\omega_{m} \int_{1 / j}^{2 / j} h_{j}(j r)^{m} r^{m-1} d r \\
& \rightarrow \operatorname{Vol}\left(B^{m}(p, 1)\right)+\operatorname{Vol}\left(\mathbb{T}^{m}\right), \\
& \left\|f_{j}\right\|_{L^{p}} \geq \operatorname{Vol}\left(B^{m}(p, 1 / j)\right) j^{p}=\omega_{m} j^{p-m} \rightarrow \infty, \quad p>m,
\end{aligned}
$$




$$
\begin{aligned}
\operatorname{Diam}\left(M_{j}\right) & =\int_{0}^{\sqrt{m} \pi} f_{j} d r \\
& =1+\int_{1 / j}^{2 / j} h_{j}(j r) d r+(\sqrt{m} \pi-2 / j) \\
& \rightarrow 1+\sqrt{m} \pi .
\end{aligned}
$$

So we see that the volume and diameter do not converge to the volume or diameter of $M_{0}$ but we do note that $g_{j} \geq g_{0}$ by construction.

Now we would like to show that $M_{j}$ converges to $M_{\infty}$ in the GH and $\mathcal{F}$ sense. We begin by constructing a sequence of maps in order to estimate the $\mathrm{GH}$ distance

$$
F_{j}: M_{\infty} \rightarrow M_{j}
$$

where

$$
F_{j}\left(\mathbb{D}^{m} \backslash \partial \mathbb{D}^{m}\right)=B_{\mathbb{T}^{m}}(p, 1 / j),
$$

is defined by scaling,

$$
F_{j}\left(\mathbb{T}^{m} \backslash B_{\mathbb{T}^{m}}(p, 3 / j)\right)=\mathbb{T}^{m} \backslash B_{\mathbb{T}^{m}}(p, 3 / j),
$$

is the identity map,

$$
F_{j}\left(B_{\mathbb{T}^{m}}(p, 3 / j) \backslash\{p\}\right)=B_{\mathbb{T}^{m}}(p, 3 / j) \backslash B_{\mathbb{T}^{m}}(p, 2 / j),
$$

is defined by radial scaling, and

$$
F_{j}(p)=q \in \partial B_{\mathbb{T}^{m}}(p, 2 / j) .
$$

By construction this map is almost onto and now we will argue that this map is almost distance preserving.

Case 1: $p_{1}, p_{2} \in \mathbb{D}^{m} \backslash \partial \mathbb{D}^{m} \subset M_{\infty}$.

Connect $F_{j}\left(p_{1}\right)$ to $F_{j}\left(p_{2}\right)$ via a straight line $\gamma \subset B_{\mathbb{T}^{m}}(p, 1 / j)$ and let $\alpha=\alpha_{1} \alpha_{2} \alpha_{3} \alpha_{4} \alpha_{5}$ be the concatenation of $\alpha_{1}, \alpha_{5} \subset B_{\mathbb{T}^{m}}(p, 1 / j), \alpha_{3} \subset$ $\partial B_{\mathbb{T}^{m}}(p, 2 / j)$ length minimizing, and $\alpha_{2}, \alpha_{4} \subset B_{\mathbb{T}^{m}}(p, 2 / j) \backslash B_{\mathbb{T}^{m}}(p, 1 / j)$ radial curves. Then we can calculate

$$
\begin{aligned}
L_{j}(\alpha) \leq & L_{j}\left(\alpha_{1}\right)+L_{j}\left(\alpha_{5}\right) \\
& \quad+2 \int_{1 / j}^{2 / j} h_{j}(j r) d r+\operatorname{Diam}\left(\partial B_{\mathbb{T}^{m}}(p, 2 / j)\right) \\
= & L_{\infty}\left(F_{j}^{-1}\left(\alpha_{1}\right)\right)+L_{\infty}\left(F_{j}^{-1}\left(\alpha_{5}\right)\right) \\
& +2 \int_{1 / j}^{2 / j} h_{j}(j r) d r+\operatorname{Diam}\left(\partial B_{\mathbb{T}^{m}}(p, 2 / j)\right) .
\end{aligned}
$$


Hence we find

$$
\begin{aligned}
d_{j}\left(F_{j}\left(p_{1}\right), F_{j}\left(p_{2}\right)\right) \leq & \min \left\{L_{j}(\gamma), \inf _{\alpha} L_{j}(\alpha)\right\} \\
\leq & d_{\infty}\left(p_{1}, p_{2}\right) \\
& \quad+2 \int_{1 / j}^{2 / j} h_{j}(j r) d r+\operatorname{Diam}\left(\partial B_{\mathbb{T}^{m}}(p, 2 / j)\right) .
\end{aligned}
$$

Let $\beta_{j}$ be the length minimizing curve with respect to $g_{j}$ connecting $F_{j}\left(p_{1}\right)$ to $F_{j}\left(p_{2}\right)$. If $\beta_{j} \subset B_{\mathbb{T}^{m}}(p, 1 / j)$ then

$$
d_{\infty}\left(p_{1}, p_{2}\right) \leq L_{\infty}\left(F_{j}^{-1}\left(\beta_{j}\right)\right)=L_{j}\left(\beta_{j}\right)=d_{j}\left(F_{j}\left(p_{1}\right), F_{j}\left(p_{2}\right)\right) .
$$

If not then we can decompose $\beta_{j}=\beta_{j}^{1} \beta_{j}^{2} \beta_{j}^{3}$ into the concatenation of $\beta_{j}^{1}, \beta_{j}^{3} \subset$ $B_{\mathbb{T}^{m}}(p, 1 / j)$ and $\beta_{j}^{2} \subset \mathbb{T}^{m} \backslash B_{\mathbb{T}^{m}}(p, 1 / j)$ so that

$$
\begin{aligned}
d_{\infty}\left(p_{1}, p_{2}\right) & \leq L_{\infty}\left(F_{j}^{-1}\left(\beta_{j}^{1}\right)\right)+L_{\infty}\left(F_{j}^{-1}\left(\beta_{j}^{3}\right)\right) \\
& \leq L_{j}\left(\beta_{j}\right)=d_{j}\left(F_{j}\left(p_{1}\right), F_{j}\left(p_{2}\right)\right) .
\end{aligned}
$$

Case 2: $p_{1}, p_{2} \in \mathbb{T}^{m} \subset M_{\infty}$.

Connect $F_{j}\left(p_{1}\right)$ to $F_{j}\left(p_{2}\right)$ via a straight line $\gamma \subset \mathbb{T}^{m} \backslash B_{\mathbb{T}^{m}}(p, 2 / j)$, if possible, and by $\alpha=\alpha_{1} \alpha_{2} \alpha_{3}$ the concatenation of $\alpha_{1}, \alpha_{3} \subset \mathbb{T}^{m} \backslash B_{\mathbb{T}^{m}}(p, 2 / j)$ and $\alpha_{2} \subset \partial B_{\mathbb{T}^{m}}(p, 2 / j)$ length minimizing. Then we can calculate

$$
\begin{aligned}
L_{j}(\alpha) & \leq L_{j}\left(\alpha_{1}\right)+L_{j}\left(\alpha_{3}\right)+2 \operatorname{Diam}\left(\partial B_{\mathbb{T}^{m}}(p, 2 / j)\right) \\
& \leq L_{\mathbb{T}^{m}}\left(\alpha_{1}\right)+L_{\mathbb{T}^{m}}\left(\alpha_{3}\right)+2 \operatorname{Diam}\left(\partial B_{\mathbb{T}^{m}}(p, 2 / j)\right) .
\end{aligned}
$$

Hence we find

$$
\begin{aligned}
d_{j}\left(F_{j}\left(p_{1}\right), F_{j}\left(p_{2}\right)\right) \leq & \min \left\{L_{j}(\gamma), \inf _{\alpha} L_{j}(\alpha)\right\} \\
\leq & d_{\infty}(p, q)+\operatorname{Diam}\left(B_{\mathbb{T}^{m}}(p, 3 / j)\right) \\
& +2 \operatorname{Diam}\left(\partial B_{\mathbb{T}^{m}}(p, 2 / j)\right)
\end{aligned}
$$

Let $\beta_{j}$ be the length minimizing curve with respect to $g_{j}$ connecting $F_{j}\left(p_{1}\right)$ to $F_{j}\left(p_{2}\right)$. Decompose $\beta_{j}=\beta_{j}^{1} \beta_{j}^{2} \beta_{j}^{3}$ into the concatenation of $\beta_{j}^{1}, \beta_{j}^{3} \subset$ $\mathbb{T}^{m} \backslash B_{\mathbb{T}^{m}}(p, 2 / j)$ and $\beta_{j}^{2} \subset B_{\mathbb{T}^{m}}(p, 2 / j)\left(\beta_{j}^{2}\right.$ and $\beta_{j}^{3}$ could be trivial if $\beta_{j} \subset$ $\left.\mathbb{T}^{m} \backslash B_{\mathbb{T}^{m}}(p, 2 / j)\right)$. Then

$$
\begin{aligned}
d_{\infty}\left(p_{1}, p_{2}\right) & \leq L_{\mathbb{T}^{m}}\left(\beta_{j}^{1}\right)+L_{\mathbb{T}^{m}}\left(\beta_{j}^{3}\right)+\operatorname{Diam}\left(B_{\mathbb{T}^{m}}(p, 3 / j)\right) \\
& \leq L_{j}\left(\beta_{j}\right)+\operatorname{Diam}\left(B_{\mathbb{T}^{m}}(p, 3 / j)\right) \\
& =d_{j}\left(F_{j}\left(p_{1}\right), F_{j}\left(p_{2}\right)\right)+\operatorname{Diam}\left(B_{\mathbb{T}^{m}}(p, 3 / j)\right) .
\end{aligned}
$$

Case 3: $p_{1} \in \mathbb{D}^{m} \backslash \partial \mathbb{D}^{m} \subset M_{\infty}, p_{2} \in \mathbb{T}^{m} \subset M_{\infty}$.

Let $\beta_{j}$ be the length minimizing curve with respect to $g_{j}$ connecting $F_{j}\left(p_{1}\right)$ to $F_{j}\left(p_{2}\right)$. Then we can decompose $\beta_{j}=\beta_{j}^{1} \beta_{j}^{2} \beta_{j}^{3}$ into the concatenation of $\beta_{j}^{1} \subset \mathbb{T}^{m} \backslash B_{\mathbb{T}^{m}}(p, 2 / j), \beta_{j}^{2} \subset B_{\mathbb{T}^{m}}(p, 2 / j) \backslash B_{\mathbb{T}^{m}}(p, 1 / j)$, and 
$\beta_{j}^{3} \subset B_{\mathbb{T}^{m}}(p, 1 / j)$. Then we can calculate

$$
\begin{aligned}
d_{\infty}(p, q) & \leq L_{\mathbb{T}^{m}}\left(\beta_{j}^{1}\right)+L_{\infty}\left(F_{j}^{-1}\left(\beta_{j}^{3}\right)\right)+\operatorname{Diam}\left(B_{\mathbb{T}^{m}}(p, 3 / j)\right) \\
& \leq L_{j}\left(\beta_{j}\right)+\operatorname{Diam}\left(B_{\mathbb{T}^{m}}(p, 3 / j)\right) \\
& =d_{j}\left(F_{j}\left(p_{1}\right), F_{j}\left(p_{2}\right)\right)+\operatorname{Diam}\left(B_{\mathbb{T}^{m}}(p, 3 / j)\right) .
\end{aligned}
$$

Similarly, if $\beta$ is a curve connecting $F_{j}\left(p_{1}\right)$ to $F_{j}\left(p_{2}\right)$, decomposed in a similar way as above, then

$$
\begin{aligned}
d_{j}\left(F_{j}\left(p_{1}\right), F_{j}\left(p_{2}\right)\right) \leq & \inf _{\beta} L_{j}(\beta) \\
= & \inf _{\beta}\left\{L_{j}\left(\beta_{j}^{1}\right)+L_{j}\left(\beta_{j}^{2}\right)+L_{j}\left(\beta_{j}^{3}\right)\right\} \\
= & \inf _{\beta}\left\{L_{\mathbb{T}^{m}}\left(\beta_{j}^{1}\right)+L_{j}\left(\beta_{j}^{2}\right)+L_{\infty}\left(F_{j}^{-1}\left(\beta_{j}^{3}\right)\right)\right\} \\
\leq & d_{\infty}\left(p_{1}, p_{2}\right)+\operatorname{Diam}\left(B_{\mathbb{T}^{m}}(p, 3 / j)\right) \\
& \quad+\operatorname{Diam}\left(\partial B_{\mathbb{T}^{m}}(p, 2 / j)\right)+\frac{1}{j} \int_{1}^{2} h_{j}(r) d r .
\end{aligned}
$$

Since all of the error terms are going to zero uniformly for $p_{1}, p_{2} \in M_{\infty}$ as $j \rightarrow \infty$ we find that $F_{j}$ is $\delta_{j}$-almost distance preserving and $\delta_{j}$-almost onto for $\delta_{j} \rightarrow 0$. Thus by Gro81b] (cf. Corollary 7.3.28 in [BBI01]) we have that

$$
M_{j} \stackrel{\mathrm{GH}}{\longrightarrow} M_{\infty} .
$$

Once $M_{j}$ has a GH limit $M_{\infty}$ then by Theorem 3.20 of SW11 we know that a subsequence converges in the $\mathcal{F}$ sense either to the 0 space or a subset of the GH limit. Now consider any point $q \in \mathbb{T}^{m} \backslash \bar{B}_{\mathbb{T}^{m}}(p, 2 / j)$ and $B_{d_{j}}(q, r) \in M_{j}$ where $r<d_{0}(p, q) \leq d_{j}(p, q)$. For $r$ chosen sufficiently small and $j$ sufficiently large this is a Euclidean ball, and so by Lemma 4.1 of Sor19, choosing possibly a smaller $r>0, B_{d_{j}}(q, r)$ converges to a Euclidean ball of radius $r$ inside the $\mathcal{F}$ limit. For $q \in B_{\mathbb{T}^{m}}(p, 1 / j)$ a similar argument implies that for $r$ small enough and $j$ large enough $B_{d_{j}}(q, r)$ converges to a Euclidean ball of radius $r$. Piecing together these Euclidean balls, we see that the intrinsic flat limit is $M_{\infty}$ with possibly one point removed. But that point is added back in when one takes the metric completion.

3.6. Conformal Tori with no $\mathbf{G H}$ nor $\mathcal{F}$ limit. Here we see an example where th $L^{p}$ norm of the conformal factor $f_{j}$ diverges for any $p>\frac{m}{\eta}, \eta>1$ and whose volume diverges. We will show that the $\mathrm{GH}$ and $\mathcal{F}$ limits are not well defined.

Example 3.6. Consider the sequence of functions on $\mathbb{T}^{m}$ which are radially defined from a point $p \in \mathbb{T}^{m}$

$$
f_{j}(r)= \begin{cases}j^{\eta} & \text { if } r \in[0,1 / j] \\ h_{j}(j r) & \text { if } r \in[1 / j, 2 / j] \\ 1 & \text { if } r \in(1 / j, \sqrt{m} \pi] .\end{cases}
$$


where $\eta>1$ and $h_{j}:[1,2] \rightarrow \mathbb{R}$ is a smooth, decreasing function so that $h_{j}(1)=j^{\eta}$ and $h_{j}(2)=1$. Then for $M_{j}=\left(\mathbb{T}^{m}, f_{j}^{2} g_{\mathbb{T}^{m}}\right)$

$$
\begin{aligned}
& \operatorname{Vol}\left(M_{j}\right) \rightarrow \infty, \\
& \operatorname{Diam}\left(M_{j}\right) \rightarrow \infty, \\
& \left\|f_{j}\right\|_{L^{p}} \rightarrow \infty, \quad p>\frac{m}{\eta},
\end{aligned}
$$

and $M_{j}$ does not converge in the $\mathcal{F}$ or $G H$ sense to any compact metric space.

Proof. We begin by computing

$$
\begin{aligned}
\operatorname{Vol}\left(M_{j}\right) & =\int_{\mathbb{T}^{m}} f_{j}^{m} d \operatorname{Vol} \\
& \geq \operatorname{Vol}\left(B^{m}(p, 1 / j)\right) j^{m \eta}+\operatorname{Vol}\left(\mathbb{T}^{m} \backslash B^{m}(p, 2 / j)\right) \\
& =j^{m(\eta-1)} \operatorname{Vol}\left(B^{m}(p, 1)\right)+\operatorname{Vol}\left(\mathbb{T}^{m} \backslash B^{m}(p, 2 / j)\right) \rightarrow \infty, \\
\left\|f_{j}\right\|_{L^{p}} & \geq \operatorname{Vol}\left(B^{m}(p, 1 / j)\right) j^{p \eta}=\omega_{m} j^{p \eta-m} \rightarrow \infty, \quad p>\frac{m}{\eta},
\end{aligned}
$$

and

$$
\operatorname{Diam}\left(M_{j}\right)=\int_{0}^{\sqrt{m} \pi} f_{j} d r \geq j^{\eta-1}+(\sqrt{m} \pi-2 / j) \rightarrow \infty .
$$

For sake of contradiction assume that $M_{j} \stackrel{\text { GH }}{\longrightarrow} M_{\infty}$ where $M_{\infty}$ is a compact metric space then the diameter $\operatorname{Diam}\left(M_{j}\right)$ must converge to $\operatorname{Diam}\left(M_{\infty}\right)$ but by (195) we find that $\operatorname{Diam}\left(M_{j}\right) \rightarrow \infty$ which is a contradiction.

Similarly, for sake of contradiction assume that $M_{j} \stackrel{\mathcal{F}}{\longrightarrow} M_{\infty}$. In Lemma 4.1 of [Sor19] it is shown that if $p_{j} \in M_{j}, M_{j} \stackrel{\mathcal{F}}{\longrightarrow} M_{\infty}$, and $B_{j}\left(p_{j}, R\right)$, viewed as an integral current space, is such that $B_{j}\left(p_{j}, R\right) \stackrel{\mathcal{F}}{\longrightarrow} H(R)$ then $H(R) \subset M_{\infty}$. Notice that $B_{j}(p, R)=B\left(p, R j^{-\eta}\right)$ which is contained in the piece of $M_{j}$ which is Euclidean space and hence $B_{j}(p, R) \stackrel{\mathcal{F}}{\longrightarrow} B(p, R) \subset M_{\infty}$. This implies that $\mathbf{M}\left(M_{\infty}\right) \geq \omega_{m} R^{m}$ for all $R>0$ which is a contradicts the fact that integral current spaces have finite volume.

3.7. Conformal Tori whose $\mathbf{G H}$ and $\mathcal{F}$ limit disagree. Here we see an example where $f_{j} \rightarrow 1$ in $L^{m}$ but such that the $L^{p}$ norm is unbounded for every $p>m$. This is the borderline case between Example 3.4 and Example 3.5. For this reason we will see that the GH limit is not the flat torus. This shows that we cannot expect the conclusion of Theorem 1.2 to hold when $f_{j}$ is unbounded. This also reinforces the fact that we will not be able to find Lipschitz or Hölder control from above on the distance function when $f_{j}$ is unbounded. Since a spline develops along the sequence a Lipschitz or Hölder bound from above cannot exist for otherwise we would contradict the main theorem of the appendix in [AB19]. 
Example 3.7. Consider the sequence of functions on $\mathbb{T}^{m}$ which are radially defined from a point $p \in \mathbb{T}^{m}$

$$
f_{j}(r)= \begin{cases}\frac{j^{\eta}}{1+\ln (j)} & \text { if } r \in\left[0,1 / j^{\eta}\right] \\ \frac{1}{r(1-\ln (r))} & \text { if } r \in\left(1 / j^{\eta}, 1 / j\right] \\ h_{j}(j r) & \text { if } r \in(1 / j, 2 / j] \\ 1 & \text { if } r \in(2 / j, \sqrt{m} \pi] .\end{cases}
$$

where $\eta>1$ and $h_{j}:[1,2] \rightarrow \mathbb{R}$ is a smooth, decreasing function so that $h_{j}(1)=\frac{j}{1+\ln (j)}$ and $h_{j}(2)=1$. Then $f_{j}$ is not bounded in $L^{p}, p>m$, but

$$
\left\|f_{j}-1\right\|_{L^{p}\left(\mathbb{T}^{m}\right)} \rightarrow 0 \text { for } p \leq m
$$

For $M_{j}=\left(\mathbb{T}^{m}, f_{j}^{2} g_{\mathbb{T}^{m}}\right)$

$$
M_{j} \stackrel{m G H}{\longrightarrow} M_{\infty}
$$

where $M_{\infty}$ is $\mathbb{T}^{m}$ with a line of length $\ln (\eta)$ attached, and

$$
M_{j} \stackrel{\mathcal{V F}}{\longrightarrow} \mathbb{T}^{m}
$$

Proof. We begin by computing

$$
\begin{aligned}
& \operatorname{Vol}\left(M_{j}\right)=\int_{\mathbb{T}^{m}} f_{j}^{m} d V \\
& =\operatorname{Vol}\left(B^{m}(p, 1 / j)\right) \frac{j^{m}}{(1+\ln (j))^{m}} \\
& \quad+\int_{B(p, 1 / j) \backslash B\left(p, 1 / j^{\eta}\right)}\left(\frac{1}{r(1-\ln (r))}\right)^{m} d V \\
& \quad+\int_{B(p, 2 / j) \backslash B(p, 1 / j)} h_{j}(j r)^{m} d V \\
& \quad+\operatorname{Vol}\left(\mathbb{T}^{m} \backslash B^{m}(p, 2 / j)\right)
\end{aligned}
$$




$$
\begin{aligned}
= & \frac{1}{(1+\ln (j))^{m}}+\operatorname{Vol}\left(\mathbb{T}^{m} \backslash B^{m}(p, 2 / j)\right) \\
& +\int_{1 / j^{\eta}}^{1 / j} \frac{\omega_{m}}{r(1-\ln (r))^{m}} d r+\int_{1 / j}^{2 / j} \omega_{m} h_{j}(j r)^{m} r^{m-1} d r \\
= & \frac{1}{(1+\ln (j))^{m}}+\operatorname{Vol}\left(\mathbb{T}^{m} \backslash B^{m}(p, 2 / j)\right) \\
& +\int_{1-\ln \left(1 / j^{\eta}\right)}^{1-\ln (1 / j)}-\frac{\omega_{m}}{u^{m}} d u+\int_{1 / j}^{2 / j} \omega_{m} h_{j}(j r)^{m} r^{m-1} d r \\
= & \frac{1}{(1+\ln (j))^{m}}+\operatorname{Vol}\left(\mathbb{T}^{m} \backslash B^{m}(p, 2 / j)\right) \\
& +\frac{\omega_{m}}{m-1}\left(\left(1-\ln \left(1 / j^{\eta}\right)\right)^{1-m}-(1-\ln (1 / j))^{1-m}\right) \\
& +\int_{1 / j}^{2 / j} \omega_{m} h_{j}(j r)^{m} r^{m-1} d r \rightarrow \operatorname{Vol}\left(\mathbb{T}^{m}\right)
\end{aligned}
$$

where we notice that

$$
\begin{aligned}
\frac{\omega_{m}\left(2^{m}-1\right)}{m j^{m}} & =\omega_{m} \int_{1 / j}^{2 / j} r^{m-1} d r \\
& \leq \int_{1 / j}^{2 / j} \omega_{m} h_{j}(j r)^{m} r^{m-1} d r \\
& \leq \omega_{m} \frac{j^{m}}{(1+\ln (j))^{m}} \int_{1 / j}^{2 / j} r^{m-1} d r=\frac{\omega_{m}\left(2^{m}-1\right)}{m(1+\ln (j))^{m}} .
\end{aligned}
$$

Now we also calculate the diameter

$$
\begin{aligned}
\operatorname{Diam}\left(M_{j}\right)= & \int_{0}^{\sqrt{m} \pi} f_{j} d r \\
= & \frac{1}{1+\ln (j)}+\int_{1 / j^{\eta}}^{1 / j} \frac{1}{r(1-\ln (r))} d r \\
& \quad+\int_{1 / j}^{2 / j} h_{j}(j r) d r+(\sqrt{m} \pi-2 / j) \\
= & \frac{1}{1+\ln (j)}+(\sqrt{m} \pi-2 / j)+\int_{1 / j}^{2 / j} h_{j}(j r) d r \\
& \quad+\ln \left(1-\ln \left(1 / j^{\eta}\right)\right)-\ln (1-\ln (1 / j)) \\
= & \frac{1}{1+\ln (j)}+(\sqrt{m} \pi-2 / j)+\int_{1 / j}^{2 / j} h_{j}(j r) d r \\
& \quad+\ln \left(\frac{1-\ln \left(1 / j^{\eta}\right)}{1-\ln (1 / j)}\right) \rightarrow \sqrt{m} \pi+\ln (\eta) .
\end{aligned}
$$

So in this case we see that the volume and diameter are controlled but the diameter does not converge to the diameter of $\mathbb{T}^{m}$. 
We also note that this example does not converge in $L^{p}$ norm for any $p>m$ since

$$
\int_{\mathbb{T}^{m}} f_{j}^{p} d \operatorname{Vol} \geq \operatorname{Vol}\left(B^{m}\left(p, \frac{1}{j^{\eta}}\right)\right) \frac{j^{\eta p}}{(1+\ln (j))^{p}}=\frac{j^{\eta(p-m)}}{(1+\ln (j))^{p}} \rightarrow \infty .
$$

Let $M_{\infty}=\mathbb{T}^{m} \sqcup[0, \ln (\eta)]$ attached at a point and consider the map

$$
F_{j}: M_{\infty} \rightarrow M_{j}
$$

defined so that $F_{j}\left(\mathbb{T}^{m}\right)=\mathbb{T}^{m} \backslash B\left(p, 1 / j^{\eta}\right)$ in a standard almost distance preserving fashion. Then $F_{j}([0, \ln (\eta)])$ is mapped onto a radial curve from p to $\partial B\left(p, 1 / j^{\eta}\right) \subset M_{j}$ so that

$$
F_{j}(r)=q, \quad q \in \partial B\left(p, 1 / j^{\eta / e^{r}}\right) .
$$

Notice that this map is almost onto. We can see that the map is almost distance preserving since for $q \in \partial B\left(p, 1 / j^{\eta / e^{r}}\right) \subset M_{j}$ where $r \leq \ln (\eta)$ we can perform a calculation similar to (215)

$$
\begin{aligned}
d_{j}(p, q) & =\int_{0}^{1 / j^{\eta / e^{r}}} f_{j} d r \\
& =\frac{1}{1+\ln (j)}+\ln \left(\frac{1-\ln \left(1 / j^{\eta}\right)}{1-\ln \left(1 / j^{\eta / e^{r}}\right)}\right) \rightarrow r .
\end{aligned}
$$

Hence $M_{j} \stackrel{\text { GH }}{\longrightarrow} M_{\infty}$ by Gro81b.

Once $M_{j}$ has a GH limit $M_{\infty}$ then by [SW11] we know that a subsequence converges in the $\mathcal{F}$ sense either to the 0 space or a subset of the $\mathrm{GH}$ limit. Since the $\mathcal{F}$ limit must have the same dimension as the sequence, the subsequence converges in the $\mathcal{F}$ sense either to the 0 space or a subset of the flat torus. Now consider any point $q \neq p$ in the flat torus and consider $B_{d_{j}}(q, r) \in M_{j}$ where $r<d_{0}(p, q) \leq d_{j}(p, q)$. For $j$ sufficiently large this is a Euclidean ball, and so by Lemma 4.1 in [Sor19], choosing possibly a smaller $r>0 B_{d_{j}}(q, r)$ converges to a Euclidean ball of radius $r$ inside the $\mathcal{F}$ limit. Piecing together these Euclidean balls, we see that the intrinsic flat limit is a flat torus with possibly one point removed. But that point is added back in when one takes the metric completion and hence we find $\mathcal{V F}$ convergence. A new proof of this limit will appear in upcoming work of the authors.

3.8. No GH Convergence. Here we build on the previous example by producing an example which does not even have a GH limit. This shows why we cannot expect to find GH convergence when we only assume a metric lower bound and volume convergence. Again, $f_{j} \rightarrow 1$ in $L^{m}$ but such that the $L^{p}$ norm is unbounded for every $p>m$ which is the borderline case between Example 3.4 and Example 3.5. This example appears to converge in the $\mathcal{V F}$ sense to a flat torus and this will be shown in a future paper by the authors. 
Example 3.8. Consider the sequence of functions on $\mathbb{T}^{m}, m \geq 2$, which are radially defined from a point $p \in \mathbb{T}^{m}$

$$
h_{j}^{p}(r)= \begin{cases}\frac{j^{\eta}}{1+\ln (j)} & \text { if } r \in\left[0,1 / j^{\eta}\right] \\ \frac{1}{r(1-\ln (r))} & \text { if } r \in\left(1 / j^{\eta}, 1 / j\right] \\ h_{j}(j r) & \text { if } r \in(1 / j, 2 / j],\end{cases}
$$

where $\eta>1$ and $h_{j}:[1,2] \rightarrow \mathbb{R}$ is a smooth, decreasing function so that $h_{j}(1)=\frac{j}{1+\ln (j)}$ and $h_{j}(2)=1$. Let $\left\{p_{1}, p_{2}, \ldots, p_{k_{j}}\right\}$, where $k_{j} \in \mathbb{N}, k_{j} \leq$ $\lfloor\sqrt{\ln (j)}\rfloor, k_{j} \rightarrow \infty$, by equally spaced points around a flat circle in $\mathbb{T}^{m}$ and define the conformal factor

$$
f_{j}(q)= \begin{cases}h_{j}^{p_{i}}(q) & \text { if } q \in B\left(p_{i}, 2 / j\right), 1 \leq i \leq k_{j} \\ 1 & \text { otherwise. }\end{cases}
$$

Then $f_{j}$ is not bounded in $L^{p}, p>m$,

$$
\left\|f_{j}-1\right\|_{L^{p}\left(\mathbb{T}^{m}\right)} \rightarrow 0 \text { for } p \leq m,
$$

but the sequence $M_{j}=\left(\mathbb{T}^{m}, f_{j}^{2} g_{\mathbb{T}^{m}}\right)$ has no GH limit.

Proof. First we verify convergence in $L^{m}$ norm

$$
\begin{aligned}
\operatorname{Vol}\left(M_{j}\right)= & \int_{\mathbb{T}^{m}} f_{j}^{m} d V o l \\
= & \sum_{i=1}^{k_{j}} \int_{B\left(p_{i}, 2 / j\right)}\left(h_{j}^{p_{i}}\right)^{m} d V o l \\
\quad & \quad+\operatorname{Vol}\left(\mathbb{T}^{m}\right)-k_{j} \operatorname{Vol}\left(B\left(p_{1}, 2 / j\right)\right) \\
= & \operatorname{Vol}\left(\mathbb{T}^{m}\right)-k_{j} \operatorname{Vol}\left(B\left(p_{1}, 2 / j\right)\right)+\frac{k_{j}}{(1+\ln (j))^{m}} \\
\quad & \quad \frac{\omega_{m} k_{j}}{m-1}\left((1+\eta \ln (j))^{1-m}-(1+\ln (j))^{1-m}\right) \\
& \quad+k_{j} \int_{1 / j}^{2 / j} \omega_{m} h_{j}(j r)^{m} r^{m-1} d r \rightarrow \operatorname{Vol}\left(\mathbb{T}^{m}\right)
\end{aligned}
$$

where we took advantage of calculation done in the proof of Examples 3.7 and the fact that $k_{j} \leq\lfloor\sqrt{\ln (j)}\rfloor$. Now we find for $q \in \partial B\left(p_{k}, 1 / j^{\eta / e^{r}}\right)$, $r \leq \ln (\eta)$ we can perform a calculation similar to (215) to find

$$
d_{j}\left(p_{k}, q\right)=\int_{0}^{1 / j^{\eta / e^{r}}} f_{j} d r \rightarrow r .
$$

So for $r=\ln (\eta)$ we find that $N(r) \geq k_{j}$, the number of balls of radius $r$ needed to cover $M_{j}$. Hence by the fact that $k_{j} \rightarrow \infty$ and Gromov's compactness theorem Gro81b we find that the sequence cannot have a GH limit. 


\section{Proof of the Main Theorem}

In this section we will use the hypotheses of Theorem 1.2 to achieve important estimates on distances, volume and diameter which will allow us to conclude with the proof of Theorem 1.1 and Theorem 1.2. Note that in each step of the proof we carefully itemize exactly which hypotheses are needed for that particular step, in this way our lemmas and propositions may be applied elsewhere.

4.1. Global Volume Convergence to Local Volume Convergence. In this subsection we prove:

Lemma 4.1. Let $g_{j}, g_{0}$ be continuous Riemannian metrics defined on the compact manifold $M$. If

$$
g_{j} \geq(1-1 / j) g_{0}
$$

and

$$
\mathrm{Vol}_{g_{j}}(M) \rightarrow \operatorname{Vol}_{g_{0}}(M)
$$

then for any measurable set $W \subset M$ we find

$$
\mathrm{Vol}_{g_{j}}(W) \rightarrow \mathrm{Vol}_{g_{0}}(W) .
$$

We start by showing that the volume of measurable sets $U \subset M$ with respect to $g_{j}$ converge to the volume with respect to $M_{0}$. Our first lemma considers the lower bound on volume for the sequence.

Lemma 4.2. Let $g_{j}, g_{0}$ be Riemannian metrics defined on the compact manifold $M$. If

$$
g_{j} \geq(1-1 / j) g_{0}
$$

then for any measurable set $U \subset M$ we find

$$
\operatorname{Vol}\left(\left(U, g_{j}\right)\right) \geq(1-1 / j)^{\frac{m}{2}} \operatorname{Vol}\left(\left(U, g_{0}\right)\right) .
$$

Proof. We note that by the assumption $g_{j} \geq g_{0}(1-1 / j)$ we immediately find the inequality for the associated measures $d v o l_{g_{j}} \geq(1-1 / j)^{m / 2} d v o l_{g_{0}}$ from which the claim follows.

We now prove Lemma 4.1

Proof. Given any measurable set $W$ and our lower bound on $g_{j}$ we can apply Lemma 4.2 to both $W$ and $M \backslash W$ to obtain

$$
\begin{aligned}
\operatorname{Vol}\left(\left(W, g_{j}\right)\right) & \geq(1-1 / j)^{\frac{m}{2}} \operatorname{Vol}\left(\left(W, g_{0}\right)\right) \\
\operatorname{Vol}\left(\left(M \backslash W, g_{j}\right)\right) & \geq(1-1 / j)^{\frac{m}{2}} \operatorname{Vol}\left(\left(M \backslash W, g_{0}\right)\right) .
\end{aligned}
$$

So we also have an upper bound on

$$
\begin{aligned}
\operatorname{Vol}\left(\left(W, g_{j}\right)\right) & =\operatorname{Vol}_{g_{j}}(M)-\operatorname{Vol}\left(\left(M \backslash W, g_{j}\right)\right) \\
& \leq \operatorname{Vol}_{g_{j}}(M)-(1-1 / j)^{\frac{m}{2}} \operatorname{Vol}\left(\left(M \backslash W, g_{0}\right)\right)
\end{aligned}
$$

Applying the volume convergence we obtain our claim. 
4.2. Convergence in $L^{\frac{m}{2}}$ norm to Volume Convergence. In this subsection we prove that convergence in $L^{\frac{m}{2}}$ norm for $m=\operatorname{dim}(M)$ combined with $g_{j} \geq(1-1 / j) g_{0}$ implies volume convergence. Note that this theorem does not require $g_{j} \leq K g_{0}$.

Lemma 4.3. Let $g_{j}, g_{0}$ be continuous Riemannian metrics defined on the compact manifold $M$. If

$$
g_{j} \geq(1-1 / j) g_{0}
$$

and

$$
\left\|g_{j}\right\|_{L_{g_{0}}^{\frac{m}{2}}(M)} \rightarrow\left\|g_{0}\right\|_{L_{g_{0}}^{\frac{m}{2}}(M)},
$$

then for any measurable set $U \subset M$ we find

$$
\mathrm{Vol}_{j}(U) \rightarrow \mathrm{Vol}_{0}(U)
$$

Proof. First notice

$$
\begin{aligned}
\left\|g_{j}\right\|_{L_{g_{0}}^{\frac{m}{2}}(U)}^{\frac{m}{2}} & =\left\|g_{j}\right\|_{L_{g_{0}}^{\frac{m}{2}}(M)}^{\frac{m}{2}}-\left\|g_{j}\right\|_{L_{g_{0}}^{\frac{m}{2}}(M \backslash U)}^{\frac{m}{2}} \\
& \leq\left\|g_{j}\right\|_{L_{g_{0}}^{\frac{m}{2}}(M)}^{\frac{m}{2}}-\left\|g_{0}\right\|_{L_{g_{0}}^{\frac{m}{2}}}^{\frac{m}{2}}(M \backslash U) \\
& \rightarrow\left\|g_{0}\right\|_{L_{g_{0}}^{\frac{m}{2}}(U)}^{\frac{m}{2}}=m^{\frac{m}{4}} \operatorname{Vol}_{0}(U) .
\end{aligned}
$$

Now by the determinant trace inequality which follows from the arithmeticgeometric mean inequality we find

$$
\operatorname{Det}_{g_{0}}\left(g_{j}\right)^{\frac{1}{m}} \leq \frac{1}{m} \operatorname{Tr}_{g_{0}}\left(g_{j}\right) \leq \frac{\left|g_{j}\right|_{g_{0}}}{m^{\frac{1}{2}}} .
$$

Here $\operatorname{Det}_{g_{0}}\left(g_{j}\right), \operatorname{Tr}_{g_{0}}\left(g_{j}\right)$ means to choose an orthonormal basis $\left\{v_{1}, \ldots, v_{m}\right\}$ for $g_{0}$ and form a symmetric, positive definite matrix by evaluating $A_{p q}=$ $g_{j}\left(v_{p}, v_{q}\right), 1 \leq p, q \leq m$ and then take the determinant or trace of that matrix, respectively. Hence if $\lambda_{1}^{2}, \ldots, \lambda_{m}^{2}$ are the eigenvalues of $g_{j}$ with respect to $g_{0}$ then,

$$
\operatorname{Tr}_{g_{0}}\left(g_{j}\right)=\sum_{i=1}^{m} \lambda_{i}^{2} \leq \sqrt{m}\left|g_{j}\right|_{g_{0}}
$$

and hence we obtain

$$
\operatorname{Det}_{g_{0}}\left(g_{j}\right) \leq \frac{\left|g_{j}\right|_{g_{0}}^{m}}{m^{\frac{m}{2}}}
$$

Using this inequality we find

$$
\begin{aligned}
\operatorname{Vol}_{j}(U) & =\int_{U} \sqrt{\operatorname{Det}_{g_{0}}\left(g_{j}\right)} d \operatorname{vol}_{g_{0}} \\
& \leq \int_{U} \frac{\left|g_{j}\right|_{g_{0}}^{\frac{m}{2}}}{m^{\frac{m}{4}}} d v o l_{g_{0}} \rightarrow \operatorname{Vol}_{0}(U) .
\end{aligned}
$$


Now we have an upper volume bound and the fact that $g_{j} \geq(1-1 / j) g_{0}$ means we can apply Lemma 4.2 for the lower bound.

4.3. Pointwise Almost Everywhere Convergence of Distances. This next theorem uses the volume convergence from above and distance controls from below to imply distances converge almost everywhere on $M \times M$. It does not require the Lipschitz bound from above and will be applied in future work of the authors.

Theorem 4.4. If $\left(M, g_{j}\right)$ are compact continuous Riemannian manifolds without boundary and $\left(M, g_{0}\right)$ is a smooth Riemannian manifold such that

$$
g_{j}(v, v) \geq g_{0}(v, v) \quad \forall v \in T M
$$

and

$$
\operatorname{Vol}_{j}(M) \rightarrow \operatorname{Vol}_{0}(M),
$$

then there exists a subsequence so that

$$
\lim _{j \rightarrow \infty} d_{j}(p, q)=d_{0}(p, q) \text { pointwise a.e. }(p, q) \in M \times M,
$$

where

$$
\begin{gathered}
d_{j}(p, q)=\inf \left\{L_{j}(C): C(0)=p, C(1)=q\right\}, \\
L_{j}(C)=\int_{0}^{1} \sqrt{g_{j}\left(C^{\prime}(s), C^{\prime}(s)\right)} d s .
\end{gathered}
$$

Proof. First we note by Lemma 2.2 that

$$
d_{0}(p, q) \leq d_{j}(p, q) \quad \forall(p, q) \in M \times M .
$$

Applying our volume convergence and Lemma 4.1 to any measurable set $U \subset M$ we have:

$$
\operatorname{Vol}_{j}(U)=\int_{U} \operatorname{Det}_{g_{0}}\left(g_{j}\right) d V_{g_{0}} \rightarrow \operatorname{Vol}_{0}(U)
$$

which will be key to the argument which follows.

Let $p, q \in M$ so that $q$ is not a cut point of $\mathrm{p}$ with respect to $g_{0}$. Let $v_{p q} \in T_{p} M$ be the smallest vector such that $\exp _{p}\left(v_{p q}\right)=q$. Note that if $q$ is a cut point then we can choose to replace it with another point which is arbitrarily close to $q$ and closer to $p$. This does not cause a problem since the set of cut points from $p$ has measure zero. Our goal is to show pointwise a.e. convergence of distances for a subsequence.

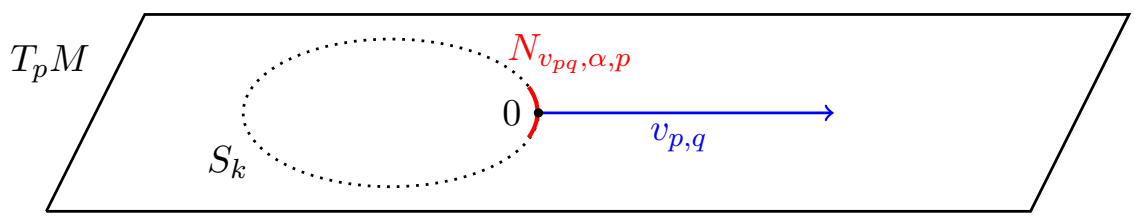

FiguRE 1. $\quad N_{v_{p q}, \alpha, p}=\left\{w \in S_{k}:|w|_{g_{0}}<\alpha\right\} \subset T_{p} M$. 
Given any $v \in T_{p} M$ we can define $S_{k} \subset T_{p} M$ be a $m-1$ sphere (or hyperplane if $k=0$ ) with constant principal curvature $k$ as a subset of $T_{p} M$, so that $0 \in S_{k}$ and $v \perp T_{0} S_{k}$. See Figure 4.3 where $v=v_{p q}$. Here we define the principal curvatures of $S_{k}$ relative to the normal vector $v$. For instance, $S_{0}=v^{\perp} \subset T_{p} M$. The parameter $k$ is important to avoid focal points later in the argument. Now for $\alpha \in(0, \infty)$ let

$$
N_{v, \alpha, p}=\left\{w \in S_{k}:|w|_{g_{0}}<\alpha\right\}
$$

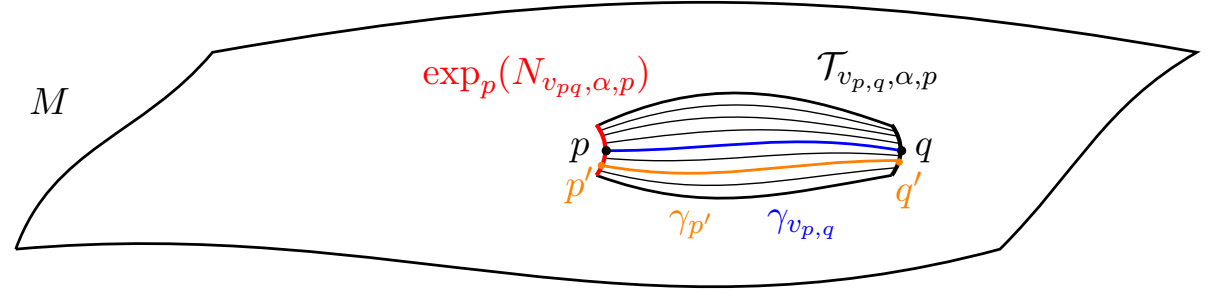

FIGURE 2. $\mathcal{T}_{v_{p q}, \alpha, p} \subset M$.

Now if $p^{\prime}=\exp _{p}(w), w \in N_{v, \alpha, p}$ and we choose $\alpha$ small enough then we can extend $v$ to $T_{p^{\prime}} M$ for all $p^{\prime} \in \exp _{p}\left(N_{v, \alpha, p}\right)$, by choosing a vector $v \in T_{p^{\prime}} M$ so that $v \perp \exp _{p}\left(S_{k}\right)$, of the same length as $v \in T_{p} M$ and so that $v$ is a continuous vector field on $\exp _{p}\left(N_{v, \alpha, p}\right)$. This allows us to define $q^{\prime}=\exp _{p^{\prime}}(v)$ as well as

$$
\gamma_{p^{\prime}}(t)=\exp _{p^{\prime}}(t v), \quad 0 \leq t \leq 1
$$

and

$$
\mathcal{T}_{v, \alpha, p}=\left\{\gamma_{p^{\prime}}(t): p^{\prime}=\exp _{p}(w), w \in N_{v, \alpha, p}, 0 \leq t \leq 1\right\} .
$$

See Figure 4.3. Now we can choose $\alpha$ small enough so that $\mathcal{T}_{v, \alpha, p}$ is foliated by $\gamma_{p^{\prime}}$ which are length minimizing with respect to $g_{0}$. If we let

$$
\exp ^{\perp}: N \exp _{p}\left(S_{k}\right) \rightarrow M
$$

be the normal exponential map where $N \exp _{p}\left(S_{k}\right)$ is the normal bundle to $\exp _{p}\left(S_{k}\right) \subset M, d \mu_{N_{v, \alpha, p}}=d \mu_{N}$ be the usual measure for $N_{v_{p q}, \alpha, p} \subset$ $T_{p} M \approx \mathbb{R}^{m}$, and $\lambda_{1}^{2}, \ldots, \lambda_{m}^{2}$ the eigenvalues of $g_{j}$ with respect to $g_{0}$ where $\lambda_{1} \leq \ldots \leq \lambda_{m}$. Let $\sqrt{h}$ be the square root of the determinant of the metric $h$ for the hypersurface $\exp \left(N_{v, \alpha, p}\right)$ in normal coordinates on $N_{v, \alpha, p}$. Let $d \exp ^{\perp}$ be the differential of the normal exponential map and $\left|d \exp ^{\perp}\right|_{g_{0}}$ be the determinant of this map evaluated in directions orthogonal to the $\gamma_{p^{\prime}}$ 
which foliate $\mathcal{T}_{v_{p q}, \alpha, p}$. Then by the coarea formula we can calculate

$$
\begin{aligned}
\operatorname{Vol}_{j}\left(\mathcal{T}_{v, \alpha, p}\right) & =\int_{\mathcal{T}_{v, \alpha}} \sqrt{\operatorname{Det}_{g_{0}}\left(g_{j}\right)} d V_{g_{0}} \\
& =\int_{N_{v, \alpha, p}} \int_{\gamma_{p^{\prime}}} \lambda_{1} \ldots \lambda_{m}\left|d \exp ^{\perp}\right|_{g_{0}} \sqrt{h} d t_{g_{0}} d \mu_{N} \\
& \geq \int_{N_{v, \alpha, p}} \int_{\gamma_{p^{\prime}}} \lambda_{1} \ldots \lambda_{m-1}\left|d \exp ^{\perp}\right|_{g_{0}} \sqrt{h} d t_{g_{j}} d \mu_{N} \\
& \geq \int_{N_{v, \alpha, p}} \int_{\gamma_{p^{\prime}}}\left|d \exp ^{\perp}\right|_{g_{0}} \sqrt{h} d t_{g_{j}} d \mu_{N} \\
& \geq \int_{N_{v, \alpha, p}} \int_{\gamma_{p^{\prime}}}\left|d \exp ^{\perp}\right|_{g_{0}} \sqrt{h} d t_{g_{0}} d \mu_{N} \\
& =\operatorname{Vol}_{0}\left(\mathcal{T}_{v, \alpha, p}\right)
\end{aligned}
$$

where we used the assumption that $g_{0} \leq g_{j}$ to deduce that

$$
\begin{aligned}
d t_{g_{0}} & =\left|\gamma^{\prime}\right|_{g_{0}} d t \leq\left|\gamma^{\prime}\right|_{g_{j}} d t=d t_{g_{j}}, \\
d t_{g_{j}} & =\left|\gamma^{\prime}\right|_{g_{j}} d t \leq \lambda_{m}\left|\gamma^{\prime}\right|_{g_{0}} d t=\lambda_{m} d t_{g_{0}} \\
\lambda_{i} & \geq 1 \quad 1 \leq i \leq m .
\end{aligned}
$$

Then since we have assumed that $\operatorname{Vol}_{j}(M) \rightarrow \operatorname{Vol}_{0}(M)$ we see by (263) that

$$
\operatorname{Vol}_{j}\left(\mathcal{T}_{v, \alpha, p}\right) \rightarrow \operatorname{Vol}_{0}\left(\mathcal{T}_{v, \alpha, p}\right)
$$

which squeezes (271) and (272) to converge to each other.

In normal coordinates we notice that $\sqrt{h}=1$ at $p$ and hence we can choose $\alpha$ small enough if needed to ensure that $\sqrt{h}>h_{0}>0$ on $N_{v, \alpha, p}$.

Now we notice that if there are no focal points between $S_{0}=v^{\perp}$ and $\gamma_{p}(t v)$ for all $0 \leq t \leq|v|_{g_{0}}$ then by construction of $\mathcal{T}_{v, \alpha, p}$ and Proposition 10.30 of O'N83 we have

$$
\left|d \exp ^{\perp}\right|_{g_{0}} \geq A_{p, q} \quad \text { on } \mathcal{T}_{v, \alpha, p},
$$

where $A_{p, q}$ is a constant which depends on the spreading of geodesics foliating $\mathcal{T}_{v, \alpha, p}$.

If it happens to be the case that there are focal points between $S_{0}=$ $v^{\perp}$ and $\gamma_{p}(t v)$ for some $0 \leq t \leq|v|_{g_{0}}$ then we can choose $k<0$ to be small enough so that the index form must be positive definite along $\gamma_{p}(t v)$ (Note that $\exp _{p}\left(S_{k}\right)$ will not have constant principal curvatures but the principal curvatures will agree with $S_{k}$ at $p$ and the difference in curvatures is determined by the metric and its Christoffel symbols in normal coordinates at $p$ ). This can be done since we know the curvature of $g_{0}$ is bounded (See the expression for the index form in ['N83] Corollary 10.27). Hence if we choose $k$ small enough we ensure that the geodesics which leave $\exp _{p}\left(S_{k}\right)$ diverge from each other enough so that they cannot meet up at a focal point 
before $q$ or even $C(p)$, the cut locus of $p$. So by Proposition 10.30 of ['N83. we know that (278) holds.

Now by combining this observation with (277) we find

$$
\begin{aligned}
\int_{N_{v, \alpha, p}} & \int_{\gamma_{p^{\prime}}}\left|d \exp ^{\perp}\right|_{g_{0}} \sqrt{h}\left(d t_{g_{j}}-d t_{g_{0}}\right) d \mu_{N} \\
& \geq A_{p, q} h_{0} \int_{N_{v, \alpha, p}} L_{j}\left(\gamma_{p^{\prime}}\right)-L_{0}\left(\gamma_{p^{\prime}}\right) d \mu_{N} \\
& \geq A_{p, q} h_{0} \int_{N_{v, \alpha, p}} d_{j}\left(p^{\prime}, q^{\prime}\right)-d_{0}\left(p^{\prime}, q^{\prime}\right) d \mu_{N} \\
& =A_{p, q} h_{0} \int_{N_{v, \alpha, p}}\left|d_{j}\left(p^{\prime}, q^{\prime}\right)-d_{0}\left(p^{\prime}, q^{\prime}\right)\right| d \mu_{N},
\end{aligned}
$$

and hence (282) converges to 0 as $j \rightarrow \infty$.

Notice that we can choose $\bar{\eta}_{2}, \bar{\eta}_{1}>0$ so that $\bar{\eta}_{1}<1<\bar{\eta}_{2}$ small enough so that for all $0<\bar{\eta}_{1}<\eta<\bar{\eta}_{2}$ the above argument works for any $\mathcal{T}_{\eta v, \alpha, p}$ and additionally we note the bound

$$
\begin{aligned}
\int_{N_{v, \alpha, p}} & \int_{\gamma_{p^{\prime}}}\left|d \exp _{p^{\prime}}^{\perp}\right|_{g_{0}} \sqrt{h}\left(d t_{g_{j}}-d t_{g_{0}}\right) d \mu_{N} \\
& \leq \operatorname{Vol}_{j}\left(\mathcal{T}_{\eta v, \alpha, p}\right)-\operatorname{Vol}_{0}\left(\mathcal{T}_{\eta v, \alpha, p}\right) \\
& \leq \operatorname{Vol}_{j}(M) \leq V_{0} .
\end{aligned}
$$

Now we can use the dominated convergence theorem to see that

$$
\int_{\bar{\eta}_{1}}^{\bar{\eta}_{2}} \int_{N_{v, \alpha, p}} \int_{\gamma_{p^{\prime}}}\left|d \exp _{p^{\prime}}^{\perp}\right|_{g_{0}} \sqrt{h}\left(d t_{g_{j}}-d t_{g_{0}}\right) d \mu_{N} d \eta \rightarrow 0 .
$$

This implies for $q_{\eta}^{\prime}=\exp _{p^{\prime}}(\eta v)$ that

$$
\int_{\bar{\eta}_{1}}^{\bar{\eta}_{2}} \int_{N_{v, \alpha, p}}\left|d_{j}\left(p^{\prime}, q_{\eta}^{\prime}\right)-d_{0}\left(p^{\prime}, q_{\eta}^{\prime}\right)\right| d \mu_{N} d \eta \rightarrow 0
$$

For sufficiently small $\bar{\tau}>0$ and any $\tau \in(-\bar{\tau}, \bar{\tau})$, we define

$$
p_{\tau}=\exp _{p}(\tau v) \text { and } p_{\tau}^{\prime} \in N_{v, \alpha, p_{\tau}}
$$

and then have

$$
q_{\eta}=q_{\eta, \tau}=\exp _{p_{\tau}}(\eta v) \text { and } q_{\eta}^{\prime}=q_{\eta, \tau}^{\prime}=\exp _{p_{\tau}^{\prime}}(\eta v)
$$

as in Figure 4.3 .

We can choose $\bar{\tau}$ small enough so that the entire argument above goes through to find

$$
\int_{-\bar{\tau}}^{\bar{\tau}} \int_{\bar{\eta}_{1}}^{\bar{\eta}_{2}} \int_{N_{v, \alpha, p_{\tau}}}\left|d_{j}\left(p_{\tau}^{\prime}, q_{\eta}^{\prime}\right)-d_{0}\left(p_{\tau}^{\prime}, q_{\eta}^{\prime}\right)\right| d \mu_{N} d \eta d \tau \rightarrow 0 .
$$

We define

$$
V_{\epsilon}=\left\{v^{\prime} \in T_{p} M:\left|v^{\prime}\right|_{g_{0}}=\left|v_{p q}\right|_{g_{0}}, g_{0}\left(v^{\prime}, v\right)>(1-\epsilon)|v|_{g_{0}}^{2}\right\} .
$$




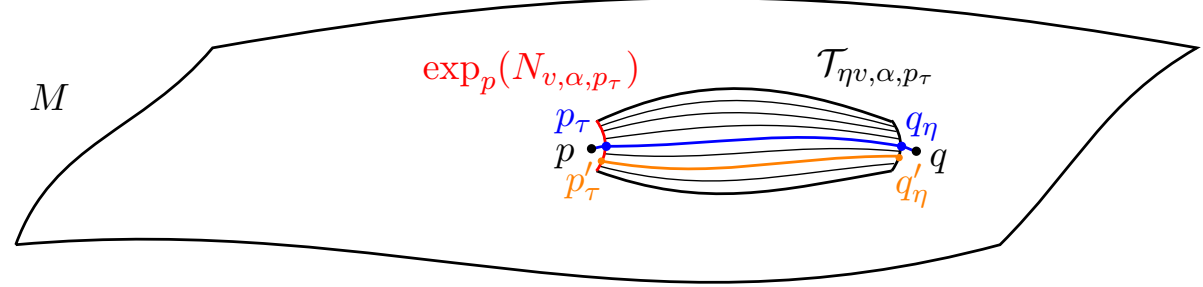

Figure 3. $\mathcal{T}_{\eta v, \alpha, p_{\tau}} \subset M$ when $\tau>0, \eta<1$, and $v=v_{p q}$.

Then again for $\epsilon$ chosen small enough we find

$$
\int_{V_{\epsilon}} \int_{-\bar{\tau}}^{\bar{\tau}} \int_{\bar{\eta}_{1}}^{\bar{\eta}_{2}} \int_{N_{v^{\prime}, \alpha, p_{\tau}}}\left|d_{j}\left(p_{\tau}^{\prime}, q_{\eta}^{\prime}\right)-d_{0}\left(p_{\tau}^{\prime}, q_{\eta}^{\prime}\right)\right| d \mu_{N} d \eta d \tau d V \rightarrow 0 .
$$

Let us define

$$
\begin{aligned}
& \mathcal{N}(p, q)= \\
& \quad\left\{\left(v^{\prime}, \tau, \eta, w\right): v^{\prime} \in V_{\epsilon}, \tau \in(-\bar{\tau}, \bar{\tau}), \eta \in\left(\bar{\eta}_{1}, \bar{\eta}_{2}\right), w \in N_{v^{\prime}, \alpha, p_{\tau}}\right\} .
\end{aligned}
$$

We claim that when $q$ is not in the cut locus of $p$ and $\epsilon, \bar{\tau}, \bar{\eta}_{i}$ are well chosen depending on $p$ and $q$ as above depending on $k, \alpha$ which were chosen so that we have (278), then the map

$$
\Phi_{p, q}: \mathcal{N}(p, q) \rightarrow M \times M \quad \Phi\left(v^{\prime}, \tau, \eta, w\right)=\left(p_{\tau}^{\prime}, q_{\eta}^{\prime}\right)
$$

as in (288)-(289) is a diffeomorphism onto its image

$$
\mathcal{U}(p, q)=\Phi(\mathcal{N}(p, q))
$$

which contains the point $(p, q)$. This can be proven by applying the inverse function theorem to $\Phi_{p, q}$ at the point $\left(v_{p, q}, 0,1,0\right)$ as follows. Take any collection of curves $v_{i}^{\prime}(t) \in V_{\epsilon}$ for $i=1 \ldots m-1$ such that $v^{\prime}(0)=v_{p q}$ and $d v_{i}^{\prime} / d t(0)$ are orthonormal and any collection of curves $w_{i}(t) \in N_{v_{p q}, \alpha, p}$ for $i=1 \ldots m-1$ such that $w_{i}(0)=0$ and $d w_{i} / d t(0)$ are orthnonormal. Then we have $2 m=(m-1)+1+1+(m-1)$ linearly independent vectors:

$$
\begin{aligned}
\left.\frac{d}{d t} \Phi_{p, q *}\left(v_{i}^{\prime}(t), 0,1,0\right)\right|_{t=0} & \in d\left(\exp _{p}\right)_{v_{p q}}\left(d v_{i}^{\prime} / d t(0)\right) \subset T_{q} M \\
\left.\frac{d}{d t} \Phi_{p, q *}\left(v_{p q}, t, 1,0\right)\right|_{t=0} & =v_{p q} \in T_{p} M \\
\left.\frac{d}{d t} \Phi_{p, q *}\left(v_{p q}, 0,1+t, 0\right)\right|_{t=0} & =\gamma_{p q}^{\prime}(1) \in T_{q} M \\
\left.\frac{d}{d t} \Phi_{p, q *}\left(v_{p q}, 0,1, w_{i}(t)\right)\right|_{t=0} & \in v_{p q}^{\perp} \subset T_{p} M
\end{aligned}
$$

by our choice of $k, \alpha$ at (278) . Thus by the inverse function theorem, possibly choosing even tighter a collection of $\epsilon, \bar{\tau}, \bar{\eta}_{i}$, we have a diffeomorphism.

Note further that pulling back $d V_{g_{0}} \times d V_{g_{0}}$ to $\mathcal{N}(p, q)$ we know that

$$
\Phi_{(p, q)}^{*}\left(d V_{g_{0}} \times d V_{g_{0}}\right)<<d V_{\mathcal{N}_{p, q}}
$$

where $<<$ denotes absolute continuity of measures. 
Combining this with (292) we see that for any $(p, q) \in M \times M$ such that $q$ is not a cut point of $p$ there exists an open set $\mathcal{U}(p, q)$ containing $(p, q)$ such that

$$
\int_{\mathcal{U}(p, q)}\left|d_{j}\left(p^{\prime}, q^{\prime}\right)-d_{0}\left(p^{\prime}, q^{\prime}\right)\right| d V_{g_{0}} \times d V_{g_{0}} \rightarrow 0 .
$$

Thus we have an open cover of $M \times M \backslash \mathcal{S}$ where

$$
\mathcal{S}=\{\{p\} \times C(p): p \in M\},
$$

where $C(p)$ is the cut locus of $p$. Since $\mathcal{S} \subset M \times M$ is a measure zero set we are allowed to proceed with our argument on $(M \times M) \backslash \mathcal{S}$. Define the continuous map

$$
\Psi:(M \times M) \backslash \mathcal{S} \rightarrow \mathbb{R}, \quad \Psi(p, q)=d_{0}(q, C(p)),
$$

so that we can define the compact sets

$$
K_{i}=\left\{(p, q) \in(M \times M) \backslash \mathcal{S}: d(q, C(p)) \geq \frac{1}{1+i}\right\} \subset(M \times M) \backslash \mathcal{S}
$$

so that $K_{i} \subset K_{i+1}$ and

$$
\bigcup_{i=1}^{\infty} K_{i}=(M \times M) \backslash \mathcal{S} .
$$

Since

$$
\left\{\mathcal{U}_{(p, q)}:(p, q) \in(M \times M) \backslash \mathcal{S}\right\},
$$

is an open over of $(M \times M) \backslash \mathcal{S}$, it is also an open cover of $K_{i}$ for each $i \in \mathbb{N}$. So we can choose a finite subcover $\left\{U_{1}, \ldots, U_{I_{1}}\right\}$ of $K_{1}$, and extend to a finite subcover of $K_{2},\left\{U_{1}, \ldots, U_{I_{1}}, \ldots U_{I_{2}}\right\}$, and continue in this way to construct a countable collection of elements of $\mathcal{U},\left\{U_{i}\right\}_{i \in \mathbb{N}}$, so that

$$
(M \times M) \backslash \mathcal{S} \subset \bigcup_{i \in \mathbb{N}} U_{i}
$$

By (302) we have for all $i \in \mathbb{N}$ :

$$
\int_{U_{i}}\left|d_{j}\left(p^{\prime}, q^{\prime}\right)-d_{0}\left(p^{\prime}, q^{\prime}\right)\right| d V_{g_{0}} \times d V_{g_{0}} \rightarrow 0 .
$$

By (309) we can choose a subsequence to find pointwise a.e. convergence of $d_{j} \rightarrow d_{0}$ on $U_{1}$. Then we can choose a further subsequence to find pointwise a.e. convergence of $d_{j} \rightarrow d_{0}$ on $U_{2}$ and continue to build a nested sequence of subsequences to obtain pointwise a.e. convergence on each $U_{i}$. By extracting a diagonal subsequence we then obtain

$$
d_{j}\left(p^{\prime}, q^{\prime}\right) \stackrel{\text { p.a.e. }}{\longrightarrow} d_{0}\left(p^{\prime}, q^{\prime}\right)
$$

for almost every $\left(p^{\prime}, q^{\prime}\right) \in M \times M$ with respect to $d V_{g_{0}} \times d V_{g_{0}}$. 
4.4. Proof of our Main Theorems. We start by proving Theorem 1.2 .

Proof. First we note that we can assume $g_{j} \geq g_{0}$ by rescaling all the metrics by $(1-1 / j)$ and we still have the same hypotheses with the volumes still converging to the volume of $g_{0}$. In the case where we assume $L^{p}$ convergence we can use Lemma 2.6, Lemma 2.7, and Lemma 4.3 to obtain volume convergence. Applying Theorem 4.4 we see that, after possibly passing to a subsequence, $d_{j}$ converge pointwise to $d_{0}$ for almost every $(p, q) \in M \times M$.

By the bi-Lipschitz bounds on $g_{j}$, we can apply Theorem 2.3 to extract a further subsequence of $M_{j}$ such that

$$
\left(M, d_{j}\right) \stackrel{\mathrm{GH}}{\longrightarrow}\left(M, d_{\infty}\right) \text { and }\left(M, d_{j}\right) \stackrel{\mathcal{F}}{\longrightarrow}\left(M, d_{\infty}\right)
$$

where $M_{\infty}=\left(M, d_{\infty}\right)$ where $d_{\infty}$ is the uniform limit of $d_{j}$. Since we already know $d_{j}$ converge pointwise to $d_{0}$ almost everywhere and both are continuous, we see that $d_{\infty}=d_{0}$.

So for every subsequence there is a further subsequence which converges

$$
\left(M, g_{j}\right) \stackrel{\mathrm{GH}}{\longrightarrow}\left(M, g_{0}\right) \text { and }\left(M, g_{j}\right) \stackrel{\mathcal{F}}{\longrightarrow}\left(M, g_{0}\right) .
$$

Thus no subsequence can converge anywhere else or fail to converge, which implies that the original sequence converges.

Applying the volume convergence hypothesis again combined with Theorem 2.4 or Theorem 4.1 we have $\left(M_{j}, g_{j}\right) \stackrel{\mathcal{V F}}{\longrightarrow}\left(M, g_{0}\right)$ and $\left(M_{j}, g_{j}\right) \stackrel{\mathrm{mGH}}{\longrightarrow}$ $\left(M, g_{0}\right)$.

We finish by noting that the proof of Theorem 1.1 follows from Theorem 1.2 ,

\section{REFERENCES}

[AB19] Brian Allen and Edward Bryden. Sobolev bounds and convergence of Riemannian manifolds. Nonlinear Anal., 185:142-169, 2019.

[AC91] Michael T. Anderson and Jeff Cheeger. Diffeomorphism finiteness for manifolds with Ricci curvature and $L^{n / 2}$-norm of curvature bounded. Geom. Funct. Anal., 1(3):231-252, 1991.

[ACT18] Clara L. Aldana, Gilles Carron, and Samuel Tapie. $a_{\infty}$ weights and compactness of conformal metrics under $L^{n / 2}$ curvature bounds. arXiv:1810.05387 [math.DG], 2018.

$\left[\mathrm{AHP}^{+} 19\right]$ B. Allen, L. Hernandez, D. Parise, A. Payne, and S. Wang. Warped tori with almost non-negative scalar curvature. Geometriae Dedicata, 200(1):153-171, 2019.

[AK00] Luigi Ambrosio and Bernd Kirchheim. Currents in metric spaces. Acta Math., 185(1):1-80, 2000.

[And90] Michael T. Anderson. Convergence and rigidity of manifolds under Ricci curvature bounds. Invent. Math., 102(2):429-445, 1990.

[And05] Michael T. Anderson. Orbifold compactness for spaces of Riemannian metrics and applications. Math. Ann., 331(4):739-778, 2005.

[AS19] Brian Allen and Christina Sormani. Contrasting various notions of convergence in geometric analysis. Pacific Journal of Mathematics, 303(1):1-46, 2019. 
[BBI01] Dmitri Burago, Yuri Burago, and Sergei Ivanov. A course in metric geometry, volume 33 of Graduate Studies in Mathematics. American Mathematical Society, Providence, RI, 2001.

[BHS08] Mario Bonk, Juha Heinonen, and Eero Saksman. Logarithmic potentials, quasiconformal flows, and Q-curvature. Duke Math. J., 142(2):197-239, 2008.

[Bre03] Simon Brendle. Global existence and convergence for a higher order flow in conformal geometry. Ann. of Math. (2), 158(1):323-343, 2003.

[CC97] Jeff Cheeger and Tobias H. Colding. On the structure of spaces with Ricci curvature bounded below. I. J. Differential Geom., 46(3):406-480, 1997.

[CGW94] S.-Y. A. Chang, M. Gursky, and T. Wolff. Lack of compactness in conformal metrics with $L^{d / 2}$ curvature. J. Geom. Anal., 4(2):143-153, 1994.

[Cha05] Sun-Yung Alice Chang. Conformal invariants and partial differential equations. Bull. Amer. Math. Soc. (N.S.), 42(3):365-393, 2005.

[Cla11] Brian Clarke. The Riemannian $L^{2}$ topology on the manifold of Riemannian metrics. Ann. Global Anal. Geom., 39(2):131-163, 2011.

[Col96] Tobias H. Colding. Shape of manifolds with positive Ricci curvature. Invent. Math., 124(1-3):175-191, 1996.

[Col97] Tobias H. Colding. Ricci curvature and volume convergence. Ann. of Math. (2), 145(3):477-501, 1997.

[Fuk87] Kenji Fukaya. Collapsing of Riemannian manifolds and eigenvalues of Laplace operator. Invent. Math., 87(3):517-547, 1987.

[Gao90] L. Zhiyong Gao. Convergence of Riemannian manifolds; Ricci and $L^{n / 2}$ curvature pinching. J. Differential Geom., 32(2):349-381, 1990.

[Gro81a] Mikhael Gromov. Groups of polynomial growth and expanding maps. Inst. Hautes Études Sci. Publ. Math., (53):53-73, 1981.

[Gro81b] Mikhael Gromov. Structures métriques pour les variétés riemanniennes, volume 1 of Textes Mathématiques [Mathematical Texts]. CEDIC, Paris, 1981. Edited by J. Lafontaine and P. Pansu.

[Gur93] Matthew J. Gursky. Compactness of conformal metrics with integral bounds on curvature. Duke Math. J., 72(2):339-367, 1993.

[HLS17] Lan-Hsuan Huang, Dan A. Lee, and Christina Sormani. Intrinsic flat stability of the positive mass theorem for graphical hypersurfaces of Euclidean space. J. Reine Angew. Math., 727:269-299, 2017.

[JL19] Jeffrey L. Jauregui and Dan A. Lee. Lower semiconituity of adm mass under intrinsic flat convergence. arXiv:1903.00916 [math.DG], 2019.

[MP17] Rostislav Matveev and Jacobus W. Portegies. Intrinsic flat and GromovHausdorff convergence of manifolds with Ricci curvature bounded below. $J$. Geom. Anal., 27(3):1855-1873, 2017.

[NnZP17] Jesús Nuñez Zimbrón and Raquel Perales. A generalized tetrahedral property. arXiv:1709.05877 [math.DG], 2017.

[O'N83] Barrett O'Neill. Semi-Riemannian geometry, volume 103 of Pure and Applied Mathematics. Academic Press, Inc. [Harcourt Brace Jovanovich, Publishers], New York, 1983. With applications to relativity.

[Pet97] Peter Petersen. Convergence theorems in Riemannian geometry. In Comparison geometry (Berkeley, CA, 1993-94), volume 30 of Math. Sci. Res. Inst. Publ., pages 167-202. Cambridge Univ. Press, Cambridge, 1997.

[PS98] Peter Petersen and Chadwick Sprouse. Integral curvature bounds, distance estimates and applications. J. Differential Geom., 50(2):269-298, 1998.

[PS17] J. Portegies and C. Sormani. Properties of the intrinsic flat distance. Algebra i Analiz, 29(3):70-143, 2017. Reprinted in St. Petersburg Math. J. 29 (2018), no. $3,475-528$.

[PW97] P. Petersen and G. Wei. Relative volume comparison with integral curvature bounds. Geom. Funct. Anal., 7(6):1031-1045, 1997. 
[PW01] Peter Petersen and Guofang Wei. Analysis and geometry on manifolds with integral Ricci curvature bounds. II. Trans. Amer. Math. Soc., 353(2):457-478, 2001.

[Sor12] Christina Sormani. How Riemannian manifolds converge. In Metric and differential geometry, volume 297 of Progr. Math., pages 91-117. Birkhäuser/Springer, Basel, 2012.

[Sor19] Christina Sormani. Intrinsic flat arzela-ascoli theorems. Communications in Analysis and Geometry, 2019.

[Stu06a] Karl-Theodor Sturm. On the geometry of metric measure spaces. I. Acta Math., 196(1):65-131, 2006.

[Stu06b] Karl-Theodor Sturm. On the geometry of metric measure spaces. II. Acta Math., 196(1):133-177, 2006.

[SW10] Christina Sormani and Stefan Wenger. Weak convergence of currents and cancellation. Calc. Var. Partial Differential Equations, 38(1-2):183-206, 2010. With an appendix by Raanan Schul and Wenger.

[SW11] Christina Sormani and Stefan Wenger. The intrinsic flat distance between Riemannian manifolds and other integral current spaces. J. Differential Geom., 87(1):117-199, 2011.

[Wan15] Yi Wang. The isoperimetric inequality and Q-curvature. Adv. Math., 281:823844, 2015.

[Yan92a] Deane Yang. Convergence of Riemannian manifolds with integral bounds on curvature. I. Ann. Sci. École Norm. Sup. (4), 25(1):77-105, 1992.

[Yan92b] Deane Yang. Convergence of Riemannian manifolds with integral bounds on curvature. II. Ann. Sci. École Norm. Sup. (4), 25(2):179-199, 1992.

[Yan92c] Deane Yang. Riemannian manifolds with small integral norm of curvature. Duke Math. J., 65(3):501-510, 1992.

UNIVERSITY OF HARTFORD

E-mail address: brianallenmath@gmail.com

CUny Graduate Center and Lehman College

E-mail address: sormanic@gmail.com 